\title{
Classification of selectors for sequences of dense sets of Baire functions
}

\author{
Alexander V. Osipov \\ Krasovskii Institute of Mathematics and Mechanics, Ural Federal University, \\ Ural State University of Economics, Yekaterinburg, Russia
}

\begin{abstract}
For a Tychonoff space $X$, we denote by $B(X)$ the space of all Baire functions on $X$ with the topology of pointwise convergence. In this paper we investigate selectors for sequences of countable dense and countable sequentially dense sets of the space $B(X)$. We give the characteristics of selection principles $S_{1}(\mathcal{P}, \mathcal{Q})$ and $S_{\text {fin }}(\mathcal{P}, \mathcal{Q})$ for $\mathcal{P}, \mathcal{Q} \in\{\mathcal{D}, \mathcal{S}, \mathcal{A}\}$, where

- $\mathcal{D}$ - the family of a countable dense subsets of $B(X)$;

- $\mathcal{S}$ - the family of a countable sequentially dense subsets of $B(X)$;

- $\mathcal{A}$ - the family of a countable 1-dense subsets of $B(X)$, through the selection principles of $X$.

Keywords: $\quad S_{1}(\mathcal{D}, \mathcal{D}), S_{\text {fin }}(\mathcal{D}, \mathcal{D}), S_{1}(\mathcal{D}, \mathcal{S}), S_{\text {fin }}(\mathcal{D}, \mathcal{S}), S_{1}(\mathcal{S}, \mathcal{D})$ $S_{\text {fin }}(\mathcal{S}, \mathcal{D}), S_{1}(\mathcal{S}, \mathcal{S}), S_{\text {fin }}(\mathcal{S}, \mathcal{S}), S_{1}\left(\mathcal{B}_{\Omega}, \mathcal{B}_{\Gamma}\right), S_{1}\left(\mathcal{B}_{\Gamma}, \mathcal{B}_{\Gamma}\right), S_{1}\left(\mathcal{B}_{\Omega}, \mathcal{B}_{\Omega}\right)$, $S_{\text {fin }}\left(\mathcal{B}_{\Omega}, \mathcal{B}_{\Omega}\right), S_{1}\left(\mathcal{B}_{\Gamma}, \mathcal{B}_{\Omega}\right), S_{1}(\mathcal{B}, \mathcal{B}), S_{1}\left(\mathcal{B}_{\Gamma}, \mathcal{B}\right)$, function spaces, selection principles, Gerlits-Nage $\gamma$ property, Baire function, $\sigma$-set, $\gamma$-set, Scheepers Diagram, sequentially separable

2000 MSC: 37F20, 26A03, 03E75, 54C35
\end{abstract}

\section{Introduction}

Many topological properties are defined or characterized in terms of the following classical selection principles. Let $\mathcal{A}$ and $\mathcal{B}$ be sets consisting of families of subsets of an infinite set $X$. Then:

Email address: OAB@list.ru (Alexander V. Osipov) 
$S_{1}(\mathcal{A}, \mathcal{B})$ is the selection hypothesis: for each sequence $\left\{A_{n}: n \in \omega\right\}$ of elements of $\mathcal{A}$ there is a sequence $\left\{b_{n}: n \in \omega\right\}$ such that for each $n, b_{n} \in A_{n}$, and $\left\{b_{n}: n \in \omega\right\}$ is an element of $\mathcal{B}$.

$S_{\text {fin }}(\mathcal{A}, \mathcal{B})$ is the selection hypothesis: for each sequence $\left\{A_{n}: n \in \omega\right\}$ of elements of $\mathcal{A}$ there is a sequence $\left\{B_{n}: n \in \omega\right\}$ of finite sets such that for each $n, B_{n} \subseteq A_{n}$, and $\bigcup_{n \in \omega} B_{n} \in \mathcal{B}$.

$U_{\text {fin }}(\mathcal{A}, \mathcal{B})$ is the selection hypothesis: whenever $\mathcal{U}_{1}, \mathcal{U}_{2}, \ldots \in \mathcal{A}$ and none contains a finite subcover, there are finite sets $\mathcal{F}_{n} \subseteq \mathcal{U}_{n}, n \in \omega$, such that $\left\{\bigcup \mathcal{F}_{n}: n \in \omega\right\} \in \mathcal{B}$.

In this paper, by cover we mean a nontrivial one, that is, $\mathcal{U}$ is a cover of $X$ if $X=\cup \mathcal{U}$ and $X \notin \mathcal{U}$.

A cover $\mathcal{U}$ of a space $X$ is:

- an $\omega$-cover if $X$ does not belong to $\mathcal{U}$ and every finite subset of $X$ is contained in a member of $\mathcal{U}$.

- a $\gamma$-cover if it is infinite and each $x \in X$ belongs to all but finitely many elements of $\mathcal{U}$.

For a topological space $X$ we denote:

- $\mathcal{O}$ - the family of countable open covers of $X$;

- $\Omega$ - the family of countable open $\omega$-covers of $X$;

- $\Gamma$ - the family of countable open $\gamma$-covers of $X$;

- $\mathcal{B}$ - the family of countable Baire (Borel for a metrizable $X$ ) covers of $X$;

- $\mathcal{B}_{\Omega}$ - the family of countable Baire $\omega$-covers of $X$;

- $\mathcal{B}_{\Gamma}$ - the family of countable Baire $\gamma$-covers of $X$.

Many equivalence hold among these properties, and the surviving ones appear in the following The extended Scheepers Diagram (where an arrow denote implication).

If $X$ is a space and $A \subseteq X$, then the sequential closure of $A$, denoted by $[A]_{\text {seq }}$, is the set of all limits of sequences from $A$. A set $D \subseteq X$ is said to be sequentially dense if $X=[D]_{\text {seq }}$. If $D$ is a countable sequentially dense subset of $X$ then $X$ call sequentially separable space.

Call $X$ strongly sequentially separable if $X$ is separable and every countable dense subset of $X$ is sequentially dense. Clearly, every strongly sequentially separable space is sequentially separable, and every sequentially separable space is separable. 


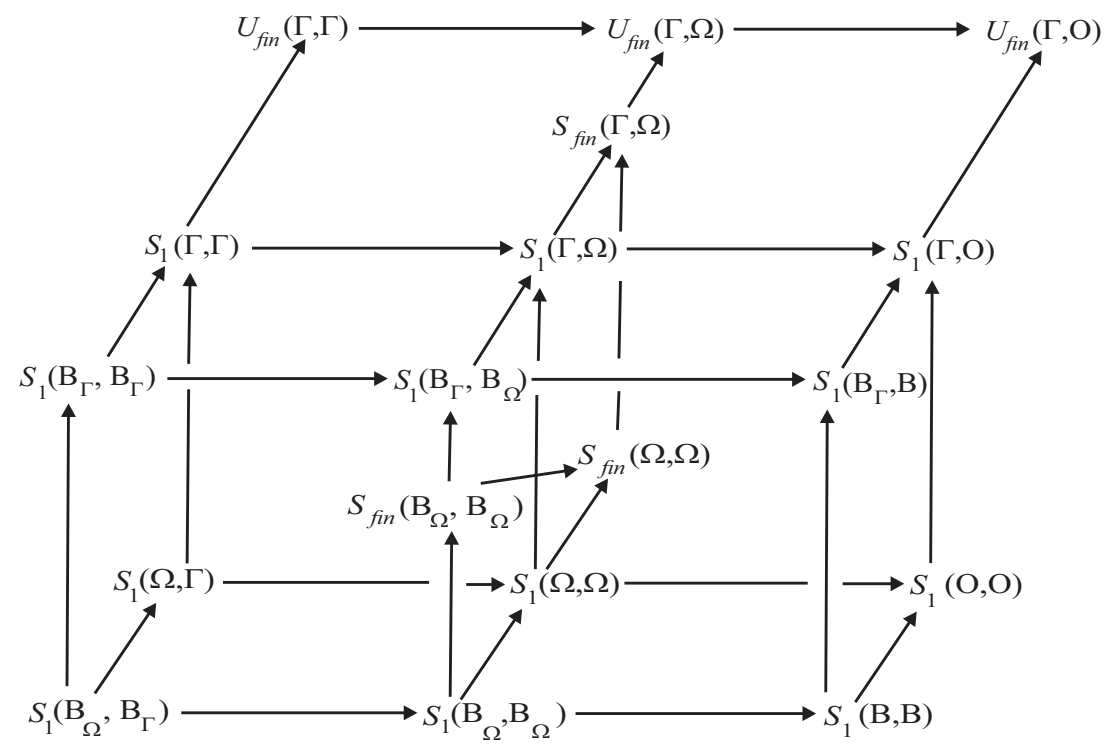

Figure 1. The extended Scheepers Diagram.

In paper [9], we investigated different selectors for sequences of dense sets of the space $C_{p}(X)$ of all real-valued continuous functions on $X$ with the topology of pointwise convergence. In this paper we investigate selectors for sequences of countable dense and countable sequentially dense sets of the space of all Baire functions, defined on a Tychonoff space $X$. We give the characteristics of selection principles $S_{1}(\mathcal{P}, \mathcal{Q})$ and $S_{\text {fin }}(\mathcal{P}, \mathcal{Q})$ for $\mathcal{P}, \mathcal{Q} \in\{\mathcal{D}$, $\mathcal{S}, \mathcal{A}\}$, where

- $\mathcal{D}$ - the family of a countable dense subsets of $B(X)$;

- $\mathcal{S}$ - the family of a countable sequentially dense subsets of $B(X)$;

- $\mathcal{A}$ - the family of a countable 1 -dense subsets of $B(X)$, through the selection principles of $X$.

\section{Main definitions and notation}

We will be denoted by

- for any $\alpha \in\left[0, \omega_{1}\right], B_{\alpha}(X)$ a set of all functions of Baire class $\alpha$, defined on a Tychonoff space $X$, provided with the pointwise convergence topology.

In particular:

- for $\alpha=0, C_{p}(X)=B_{0}(X)$ a set of all real-valued continuous functions $C(X)$ defined on a Tychonoff space $X$. 
- for $\alpha=1, B_{1}(X)$ a set of all first Baire class functions $B_{1}(X)$ i.e., pointwise limits of continuous functions, defined on a Tychonoff space $X$.

- for $\alpha=\omega_{1}, B(X)=B_{\omega_{1}}(X)$ a set of all Baire functions, defined on a Tychonoff space $X$. If $X$ is metrizable space, then $B(X)$ be called a space of Borel functions.

Basic open sets of $B_{\alpha}(X)$ are of the form

$\left[x_{1}, \ldots, x_{k}, U_{1}, \ldots, U_{k}\right]=\left\{f \in B_{\alpha}(X): f\left(x_{i}\right) \in U_{i}, i=1, \ldots, k\right\}$, where each $x_{i} \in X$ and each $U_{i}$ is a non-empty open subset of $\mathbb{R}$. Sometimes we will write the basic neighborhood of the point $f$ as $<f, A, \epsilon>$ where $<f, A, \epsilon>:=\left\{g \in B_{\alpha}(X):|f(x)-g(x)|<\epsilon \forall x \in A\right\}, A$ is a finite subset of $X$ and $\epsilon>0$.

Let $X$ be a topological space, and $x \in X$. A subset $A$ of $X$ converges to $x, x=\lim A$, if $A$ is infinite, $x \notin A$, and for each neighborhood $U$ of $x$, $A \backslash U$ is finite. Consider the following collection: $\Omega_{x}=\{A \subseteq X: x \in \bar{A} \backslash A\}$ and $\Gamma_{x}=\left\{A \subseteq X:|A|=\aleph_{0}\right.$ and $\left.x=\lim A\right\}$. We write $\Pi\left(\mathcal{A}_{x}, \mathcal{B}_{x}\right)$ without specifying $x$, we mean $(\forall x) \Pi\left(\mathcal{A}_{x}, \mathcal{B}_{x}\right)$.

- A space $X$ has countable fan tightness (Arhangel'skii's countable fan tightness), if $X \models S_{\text {fin }}\left(\Omega_{x}, \Omega_{x}\right)[2]$.

- A space $X$ has countable strong fan tightness (Sakai's countable strong fan tightness), if $X \models S_{1}\left(\Omega_{x}, \Omega_{x}\right)$ [12].

- A space $X$ has countable selectively sequentially fan tightness (Arhangel'skii's property $\left.\alpha_{4}\right)$, if $X \models S_{\text {fin }}\left(\Gamma_{x}, \Gamma_{x}\right)[1]$.

- A space $X$ has countable strong selectively sequentially fan tightness (Arhangel'skii's property $\alpha_{2}$ ), if $X \models S_{1}\left(\Gamma_{x}, \Gamma_{x}\right)$ [1].

- A space $X$ has strictly Fréchet-Urysohn at $x$, if $X \models S_{1}\left(\Omega_{x}, \Gamma_{x}\right)$ [13].

- A space $X$ has almost strictly Fréchet-Urysohn at $x$, if $X \models S_{\text {fin }}\left(\Omega_{x}, \Gamma_{x}\right)$. [14].

- A space $X$ has the weak sequence selection property, if $X \models S_{1}\left(\Gamma_{x}, \Omega_{x}\right)$

- A space $X$ has the sequence selection property, if $X \models S_{f i n}\left(\Gamma_{x}, \Omega_{x}\right)$.

The following implications hold

$$
\begin{gathered}
S_{1}\left(\Gamma_{x}, \Gamma_{x}\right) \Rightarrow S_{f i n}\left(\Gamma_{x}, \Gamma_{x}\right) \Rightarrow S_{1}\left(\Gamma_{x}, \Omega_{x}\right) \Rightarrow \underset{\text { fin }}{\Uparrow}\left(\Gamma_{x}, \Omega_{x}\right) \\
S_{1}\left(\Omega_{x}, \Gamma_{x}\right) \Rightarrow S_{f i n}\left(\Omega_{x}, \Gamma_{x}\right)
\end{gathered}
$$


We write $\Pi\left(\mathcal{A}, \mathcal{B}_{x}\right)$ without specifying $x$, we mean $(\forall x) \Pi\left(\mathcal{A}, \mathcal{B}_{x}\right)$.

- A space $X$ has countable fan tightness with respect to countable dense subspaces, if $X \models S_{\text {fin }}\left(\mathcal{D}, \Omega_{x}\right)([3])$.

- A space $X$ has countable strong fan tightness with respect to countable dense subspaces, if $X \models S_{1}\left(\mathcal{D}, \Omega_{x}\right)([3])$.

- A space $X$ has almost strictly Fréchet-Urysohn at $x$ with respect to countable dense subspaces, if $X \models S_{\text {fin }}\left(\mathcal{D}, \Gamma_{x}\right)$.

- A space $X$ has strictly Fréchet-Urysohn at $x$ with respect to countable dense subspaces, if $X \models S_{1}\left(\mathcal{D}, \Gamma_{x}\right)$.

- A space $X$ has countable selectively sequentially fan tightness with respect to countable dense subspaces, if $X \models S_{\text {fin }}\left(\mathcal{S}, \Gamma_{x}\right)$.

- A space $X$ has countable strong selectively sequentially fan tightness with respect to countable dense subspaces, if $X \models S_{1}\left(\mathcal{S}, \Gamma_{x}\right)$.

- A space $X$ has the sequence selection property with respect to countable dense subspaces, if $X \models S_{\text {fin }}\left(\mathcal{S}, \Omega_{x}\right)$.

- A space $X$ has the weak sequence selection property with respect to countable dense subspaces, if $X \models S_{1}\left(\mathcal{S}, \Omega_{x}\right)$.

The following implications hold

$$
\begin{aligned}
& S_{1}\left(\mathcal{S}, \Gamma_{x}\right) \Rightarrow S_{f i n}\left(\mathcal{S}, \Gamma_{x}\right) \Rightarrow S_{1}\left(\mathcal{S}, \Omega_{x}\right) \Rightarrow S_{f i n}\left(\mathcal{S}, \Omega_{x}\right) \\
& \begin{array}{c}
\Uparrow \\
S_{1}\left(\mathcal{D}, \Gamma_{x}\right) \Rightarrow S_{\text {fin }}\left(\mathcal{D}, \Gamma_{x}\right)
\end{array} \Rightarrow S_{1}\left(\mathcal{D}, \Omega_{x}\right) \Rightarrow \stackrel{\Uparrow}{\Uparrow} S_{\text {fin }}\left(\mathcal{D}, \Omega_{x}\right)
\end{aligned}
$$

The following implications hold for countable dense and countable sequentially dense subsets of topological space.

$$
\begin{aligned}
& S_{1}(\mathcal{S}, \mathcal{S}) \Rightarrow S_{\text {fin }}(\mathcal{S}, \mathcal{S}) \Rightarrow S_{1}(\mathcal{S}, \mathcal{D}) \Rightarrow S_{\text {fin }}(\mathcal{S}, \mathcal{D}) \\
& \Uparrow \quad \Uparrow \quad \Uparrow \quad \text { 介 } \\
& S_{1}(\mathcal{D}, \mathcal{S}) \Rightarrow S_{\text {fin }}(\mathcal{D}, \mathcal{S}) \Rightarrow S_{1}(\mathcal{D}, \mathcal{D}) \Rightarrow S_{\text {fin }}(\mathcal{D}, \mathcal{D})
\end{aligned}
$$

We recall that a subset of $X$ that is the complete preimage of zero for a certain function from $C(X)$ is called a zero-set. A subset $O \subseteq X$ is called a cozero-set (or functionally open) of $X$ if $X \backslash O$ is a zero-set.

Recall that the $i$-weight $i w(X)$ of a space $X$ is the smallest infinite cardinal number $\tau$ such that $X$ can be mapped by a one-to-one continuous mapping onto a Tychonoff space of the weight not greater than $\tau$. 
Theorem 2.1. (Noble's Theorem in [Y]) Let $X$ be a Tychonoff space. A space $C_{p}(X)$ is separable if and only if iw $(X)=\aleph_{0}$.

Theorem 2.2. (Pestrjkov's Theorem in [11]). Let $X$ be a Tychonoff space and $0 \leq \alpha \leq \omega_{1}$. A space $B_{\alpha}(X)$ is separable if and only if $i w(X)=\aleph_{0}$.

Definition 2.3. A space $X$ has $O$-property $(X \models O)$, if there exist a bijection $\varphi: X \mapsto Y$ from a space $X$ onto a separable metrizable space $Y$, such that

1. $\varphi^{-1}(U)$ - Baire set of $X$ for any open set $U$ of $Y$;

2. $\varphi(T)-F_{\sigma}$-set of $Y$ for any Baire set $T$ of $X$.

By Corollary 4.4, Corollary 4.5 and Corollary 4.6 in [8], we have

Theorem 2.4. (Osipov) For each Tychonoff space $X$ the following are equivalent:

1. $B(X)$ is sequentially separable;

2. $X \models O$;

3. $\exists$ a Baire isomorphism $f: X \mapsto M$ of class $\alpha$ from a space $X$ onto a $\sigma$-set $M$ for some $\alpha \in\left(1, \omega_{1}\right)$.

Recall that the cardinal $\mathfrak{p}$ is the smallest cardinal so that there is a collection of $\mathfrak{p}$ many subsets of the natural numbers with the strong finite intersection property but no infinite pseudo-intersection. Note that $\omega_{1} \leq \mathfrak{p} \leq \mathfrak{c}$.

For $f, g \in \mathbb{N}^{\mathbb{N}}$, let $f \leq^{*} g$ if $f(n) \leq g(n)$ for all but finitely many $n$. $\mathfrak{b}$ is the minimal cardinality of a $\leq^{*}$-unbounded subset of $\mathbb{N}^{\mathbb{N}}$. A set $B \subset[\mathbb{N}]^{\infty}$ is unbounded if the set of all increasing enumerations of elements of $B$ is unbounded in $\mathbb{N}^{\mathbb{N}}$, with respect to $\leq^{*}$. It follows that $|B| \geq \mathfrak{b}$. A subset $S$ of the real line is called a $Q$-set if each one of its subsets is a $G_{\delta}$. The cardinal $\mathfrak{q}$ is the smallest cardinal so that for any $\kappa<\mathfrak{q}$ there is a $Q$-set of size $\kappa$. (See [5] for more on small cardinals including $\mathfrak{p}$ ).

For a collection $\mathcal{J}$ of spaces, let non $(\mathcal{J})$ denote the minimal cardinality for a space which is not a member of $\mathcal{J}$. 


\section{3. $B(X) \models S_{1}(\mathcal{D}, \mathcal{D})$}

Theorem 3.1. For a Tychonoff space $X$, the following are equivalent:

1. $B(X) \models S_{1}\left(\Omega_{x}, \Omega_{x}\right)$;

2. $X \models S_{1}\left(\mathcal{B}_{\Omega}, \mathcal{B}_{\Omega}\right)$.

Proof. We prove similarly the proof of Theorem 1 in [12] for $C_{p}(X)$.

$(1) \Rightarrow(2)$. Let $\left\{\mathcal{U}_{n}\right\}_{n \in \omega}$ be a sequence of Baire $\omega$-covers of $X$. We set $A_{n}=\left\{f \in B(X): f \uparrow(X \backslash U)=0\right.$ for some $\left.U \in \mathcal{U}_{n}\right\}$. It is not difficult to see that each $A_{n}$ is dense in $B(X)$ since each $\mathcal{U}_{n}$ is an $\omega$-cover of $X$ and $X$ is Tychonoff. Let $f$ be the constant function to 1 . By the assumption there exist $f_{n} \in A_{n}$ such that $f \in \overline{\left\{f_{n}: n \in \omega\right\}}$. For each $f_{n}$ we take $U_{n} \in \mathcal{U}_{n}$ such that $f_{n}\left\lceil\left(X \backslash U_{n}\right)=0\right.$. Set $\mathcal{U}=\left\{U_{n}: n \in \omega\right\}$. For each finite subset $\left\{x_{1}, \ldots, x_{k}\right\}$ of $X$ we consider the basic open neighborhood of $f\left[x_{1}, \ldots, x_{k} ; W, \ldots, W\right]$, where $W=(0,2)$. Note that $\left[x_{1}, \ldots, x_{k} ; W, \ldots, W\right]$ contains some $f_{n}$. This means $\left\{x_{1}, \ldots, x_{k}\right\} \subset U_{n}$. Consequently $\mathcal{U}$ is an $\omega$-cover of $X$.

$(2) \Rightarrow(1)$. Let $f \in \bigcap_{n} \overline{A_{n}}$, where $A_{n}$ is a subset of $B(X)$. Since $B(X)$ is homogeneous, we may think that $f$ is the constant function to the zero. We set $\mathcal{U}_{n}=\left\{g^{-1}(-1 / n, 1 / n): g \in A_{n}\right\}$ for each $n \in \omega$. For each $n \in \omega$ and each finite subset $\left\{x_{1}, x_{2}, \ldots, x_{k}\right\}$ of $X$ a neighborhood $\left[x_{1}, \ldots, x_{k} ; W, \ldots, W\right]$ of $f$, where $W=(-1 / n, 1 / n)$, contains some $g \in A_{n}$. This means that each $\mathcal{U}_{n}$ is the Baire $\omega$-cover of $X$. In case the set $M=\left\{n \in \omega: X \in \mathcal{U}_{n}\right\}$ is infinite, choose $g_{m} \in A_{m} m \in M$ so that $g^{-1}(-1 / m, 1 / m)=X$, then $g_{m} \mapsto f$. So we may assume that there exists $n \in \omega$ such that for each $m \geq n$ and $g \in A_{m}$ $g^{-1}(-1 / m, 1 / m)$ is not $X$. For the sequence $\left\{\mathcal{U}_{m}: m \geq n\right\}$ of Baire $\omega$-covers there exist $f_{m} \in A_{m}$ such that $\mathcal{U}=\left\{f_{m}^{-1}(-1 / m, 1 / m): m \geq n\right\}$ is Baire $\omega$-cover of $X$. Let $\left[x_{1}, \ldots, x_{k} ; W, \ldots, W\right]$ be any basic open neighborhood of $f$, where $W=(-\epsilon, \epsilon), \epsilon>0$. There exists $m \geq n$ such that $\left\{x_{1}, \ldots, x_{k}\right\} \subset$ $f_{m}^{-1}(-1 / m, 1 / m)$ and $1 / m<\epsilon$. This means $f \in \overline{\left\{f_{m}: m \geq n\right\}}$.

Theorem 3.2. For a Tychonoff space $X$, the following are equivalent:

1. $B(X) \models S_{1}(\mathcal{D}, \mathcal{D})$ and it is separable;

2. $X \models S_{1}\left(\mathcal{B}_{\Omega}, \mathcal{B}_{\Omega}\right)$ and iw $(X)=\aleph_{0}$;

3. $B(X) \models S_{1}\left(\Omega_{x}, \Omega_{x}\right)$ and it is separable;

4. $B(X) \models S_{1}\left(\mathcal{D}, \Omega_{x}\right)$ and it is separable. 
Proof. (1) $\Rightarrow(2)$. Let $X$ be a Tychonoff space satisfying the hypotheses. By Pestrjkov's Theorem 2.2, $i w(X)=\aleph_{0}$. Consider a condensation (one-to-one continuous mapping) $g: X \mapsto Z$ from a space $X$ onto a separable metrizable space $Z$. Let $\beta$ be a countable base of $Z$. Denote $g^{-1}(\beta):=\left\{g^{-1}(B): B \in\right.$ $\beta\}$.

Let $\left\{\mathcal{B}_{i}\right\}_{i \in \omega}$ be a sequence of countable Borel $\omega$-covers of $X$ where $\mathcal{B}_{i}=$ $\left\{W_{i}^{j}\right\}_{j \in \omega}$ for each $i \in \omega$.

Consider a topology $\tau$ generated by the family $\mathcal{P}=\left\{W_{i}^{j} \cap A_{s}: i, j \in \omega\right.$ and $\left.A_{s} \in g^{-1}(\beta)\right\} \bigcup\left\{\left(X \backslash W_{i}^{j}\right) \cap A_{s}: i, j \in \omega\right.$ and $\left.A_{s} \in g^{-1}(\beta)\right\}$.

Note that if $\chi_{P}$ is a characteristic function of $P$ for each $P \in \mathcal{P}$, then a diagonal mapping $\varphi=\Delta_{P \in \mathcal{P}} \chi_{P}: X \mapsto 2^{\omega}$ is a Baire bijection onto $Z=$ $\varphi(X) \subset 2^{\omega}$. A space $Z=(X, \tau)$ is a separable metrizable space. Note that $\varphi\left(\mathcal{B}_{i}\right)=\left\{\varphi(B): B \in \mathcal{B}_{i}\right\}$ is countable open $\omega$-cover of $Z$ for each $i \in \omega$. Since $B(\varphi(X))=B(Z)$ is a dense subset of $B(X)$, then $B(Z)$ also has property $S_{1}(\mathcal{D}, \mathcal{D})$. Since $C_{p}(Z)$ is a dense subset of $B(Z), C_{p}(Z)$ also has property $S_{1}(\mathcal{D}, \mathcal{D})$.

By Theorem 3.3 in [8], the space $Z$ has property $S_{1}(\Omega, \Omega)$. It follows that there is a sequence $\left\{W_{i}^{j(i)}\right\}_{i \in \omega}$ such that $W_{i}^{j(i)} \in \mathcal{B}_{i}$ and $\left\{\varphi\left(W_{i}^{j(i)}\right): i \in \omega\right\}$ is open $\omega$-cover of $Z$. It follows that $\left\{W_{i}^{j(i)}: i \in \omega\right\}$ is Baire $\omega$-cover of $X$.

$(2) \Rightarrow(1)$. Assume that $X$ has property $S_{1}\left(B_{\Omega}, B_{\Omega}\right)$ and $i w(X)=\aleph_{0}$. By Pestrjkov's Theorem, $B(X)$ is a separable space.

Let $\left\{D_{i}\right\}_{i \in \omega}$ be a sequence countable dense subsets of $B(X)$. We claim that for any $f \in B(X)$ there is a sequence $\left\{f_{k}\right\} \subset B(X)$ such that $f_{k} \in D_{k}$ for each $k \in \omega$ and $f \in \overline{\left\{f_{k}: k \in \omega\right\}}$. Without loss of generality we can assume $f=\mathbf{0}$. Denote $W_{i}^{k}=\left\{x \in X:-\frac{1}{k}<f_{i}^{k}(x)<\frac{1}{k}\right\}$ for each $f_{i}^{k} \in D_{k}=$ $\left\{f_{i}^{k}: i \in \omega\right\}$ and $k \in \omega$.

If for each $j \in \omega$ there is $k(j)$ such that $W_{i(j)}^{k(j)}=X$, then a sequence $f_{k(j)}=f_{i(j)}^{k(j)}$ uniform convergence to $f$ and, hence, $f \in \overline{\left\{f_{k(j)}: j \in \omega\right\}}$.

We can assume that $W_{i}^{k} \neq X$ for any $k, i \in \omega$.

(a). $\left\{W_{i}^{k}\right\}_{i \in \omega}$ a sequence of Baire sets of $X$.

(b). For each $k \in \omega,\left\{W_{i}^{k}: i \in \omega\right\}$ is a $\omega$-cover of $X$.

By (2), $X$ has property $S_{1}\left(\mathcal{B}_{\Omega}, \mathcal{B}_{\Omega}\right)$, hence, there is a sequence $\left\{W_{i(k)}^{k}\right\}_{k \in \omega}$ such that $W_{i(k)}^{k} \in\left\{W_{i}^{k}: i \in \omega\right\}$ for each $k \in \omega$ and $\left\{W_{i(k)}^{k}: k \in \omega\right\}$ is a $\omega$-cover of $X$.

Consider $\left\{f_{i(k)}^{k}\right\}_{k \in \omega}$ and we claim that $f \in \overline{\left\{f_{i(k)}^{k}: k \in \omega\right\}}$. Let $K$ be a finite subset of $X, \epsilon>0$ and $U=<f, K, \epsilon>$ be a base neighborhood of $f$, then there is $k_{0} \in \omega$ such that $\frac{1}{k_{0}}<\epsilon$ and $K \subset W_{i\left(k_{0}\right)}^{k_{0}}$. It follows that 
$f_{i\left(k_{0}\right)}^{k_{0}} \in U$

Let $D=\left\{d_{n}: n \in \omega\right\}$ be a dense subspace of $B(X)$. Given a sequence $\left\{D_{i}\right\}_{i \in \omega}$ of dense subspace of $B(X)$, enumerate it as $\left\{D_{n, m}: n, m \in \omega\right\}$. For each $n \in \omega$, pick $d_{n, m} \in D_{n, m}$ so that $d_{n} \in \overline{\left\{d_{n, m}: m \in \omega\right\}}$. Then $\left\{d_{n, m}: m, n \in \omega\right\}$ is dense in $B(X)$.

$(2) \Leftrightarrow(3)$. By Theorem 3.1 and Theorem 2.2 .

$(1) \Leftrightarrow(4)$. By Proposition 52 in [3] for countable dense subsets of $B(X)$.

\section{4. $B(X) \models S_{\text {fin }}(\mathcal{D}, \mathcal{D})$}

Theorem 4.1. For a Tychonoff space $X$, the following are equivalent:

1. $B(X) \models S_{f i n}\left(\Omega_{x}, \Omega_{x}\right)$;

2. $X \models S_{\text {fin }}\left(\mathcal{B}_{\Omega}, \mathcal{B}_{\Omega}\right)$.

Proof. (1) $\Rightarrow(2)$. Let $\left\{\mathcal{U}_{n}: n \in \omega\right\}$ be a sequence of Baire $\omega$-covers of $X$. We set $A_{n}=\left\{f \in B(X): f \uparrow(X \backslash U)=0\right.$ for some $\left.U \in \mathcal{U}_{n}\right\}$. It is not difficult to see that each $A_{n}$ is dense in $B(X)$ since each $\mathcal{U}_{n}$ is an $\omega$-cover of $X$ and $X$ is Tychonoff. Let $f$ be the constant function to 1 . By the assumption there exist $F_{n} \subset A_{n}$ such that $f \in \bar{\bigcup}\left\{F_{n}: n \in \omega\right\}$. For each $F_{n}$ we take $W_{n}=\left\{U_{n}^{j}\right\}_{j=1}^{k(n)} \subset \mathcal{U}_{n}$ such that $f_{n}^{j} \uparrow\left(X \backslash U_{n}^{j}\right)=0$ for $f_{n}^{j} \in F_{n}$ and $U_{n}^{j} \in W_{n}$ where $j=1, \ldots, k(n)$.

Set $\mathcal{U}=\bigcup_{n \in \omega} W_{n}$. For each finite subset $\left\{x_{1}, \ldots, x_{k}\right\}$ of $X$ we consider the basic open neighborhood of $f\left[x_{1}, \ldots, x_{k} ; W, \ldots, W\right]$, where $W=(0,2)$. Note that $\left[x_{1}, \ldots, x_{k} ; W, \ldots, W\right]$ contains some $f_{n}^{j(n)}$. This means $\left\{x_{1}, \ldots, x_{k}\right\} \subset$ $U_{n}^{j(n)}$. Consequently $\mathcal{U}$ is an $\omega$-cover of $X$.

$(2) \Rightarrow(1)$. Let $f \in \bigcap \overline{A_{n}}$, where $A_{n}$ is a subset of $B(X)$. Since $B(X)$ is homogeneous, we may think that $f$ is the constant function to the zero. We set $\mathcal{U}_{n}=\left\{g^{-1}(-1 / n, 1 / n): g \in A_{n}\right\}$ for each $n \in \omega$. For each $n \in \omega$ and each finite subset $\left\{x_{1}, \ldots, x_{k}\right\}$ of $X$ a neighborhood $\left[x_{1}, \ldots, x_{k} ; W, \ldots, W\right]$ of $f$, where $W=(-1 / n, 1 / n)$, contains some $g \in A_{n}$. This means that each $\mathcal{U}_{n}$ is the Baire $\omega$-cover of $X$. In case the set $M=\left\{n \in \omega: X \in \mathcal{U}_{n}\right\}$ is infinite, choose $g_{m} \in A_{m} m \in M$ so that $g^{-1}(-1 / m, 1 / m)=X$, then $g_{m} \mapsto f$. So we may assume that there exists $n \in \omega$ such that for each $m \geq n$ and $g \in A_{m}$ $g^{-1}(-1 / m, 1 / m)$ is not $X$. For the sequence $\left\{\mathcal{U}_{m}: m \geq n\right\}$ of Baire $\omega$ there exist $F_{m}=\left\{f_{m, 1}, \ldots, f_{m, k(m)}\right\} \subset A_{m}$ such that 


$$
\mathcal{U}=\bigcup_{m \geq n}\left\{f_{m, 1}^{-1}(-1 / m, 1 / m), \ldots, f_{m, k(m)}^{-1}(-1 / m, 1 / m): i=1, \ldots, k(m)\right\}
$$

is a Baire $\omega$-cover of $X$. Let $\left[x_{1}, \ldots, x_{k} ; W, \ldots, W\right]$ be any basic open neighborhood of $f$, where $W=(-\epsilon, \epsilon), \epsilon>0$. There exists $m \geq n$ and $j \in$ $\{1, \ldots, k(m)\}$ such that $\left\{x_{1}, \ldots, x_{k}\right\} \subset f_{m, j}^{-1}(-1 / m, 1 / m)$ and $1 / m<\epsilon$. This means $f \in \overline{\bigcup\left\{F_{m}: m \geq n\right\}}$.

Theorem 4.2. For a Tychonoff space $X$, the following are equivalent:

1. $B(X) \models S_{\text {fin }}(\mathcal{D}, \mathcal{D})$ and it is separable;

2. $X \models S_{\text {fin }}\left(\mathcal{B}_{\Omega}, \mathcal{B}_{\Omega}\right)$ and $i w(X)=\aleph_{0}$;

3. $B(X) \models S_{f i n}\left(\Omega_{x}, \Omega_{x}\right)$ and it is separable;

4. $B(X) \models S_{\text {fin }}\left(\mathcal{D}, \Omega_{x}\right)$ and it is separable.

Proof. $(1) \Rightarrow(2)$. Let $X$ be a Tychonoff space satisfying the hypotheses. By Pestrjkov's Theorem 2.2, $i w(X)=\aleph_{0}$. Consider a condensation (one-to-one continuous mapping) $g: X \mapsto Z$ from a space $X$ onto a separable metrizable space $Z$. Let $\beta$ be a countable base of $Z$. Denote $g^{-1}(\beta):=\left\{g^{-1}(B): B \in\right.$ $\beta\}$.

Let $\left\{\mathcal{B}_{i}\right\}_{i \in \omega}$ be a sequence of countable Baire $\omega$-covers of $X$ where $\mathcal{B}_{i}=$ $\left\{W_{i}^{j}: j \in \omega\right\}$ for each $i \in \omega$.

Consider a topology $\tau$ generated by the family $\mathcal{P}=\left\{W_{i}^{j} \cap A_{s}: i, j \in \omega\right.$ and $\left.A_{s} \in g^{-1}(\beta)\right\} \bigcup\left\{\left(X \backslash W_{i}^{j}\right) \cap A_{s}: i, j \in \omega\right.$ and $\left.A_{s} \in g^{-1}(\beta)\right\}$.

A space $Z=(X, \tau)$ is a separable metrizable space. Note that $\left\{\mathcal{B}_{i}\right\}$ is countable open $\omega$-cover of $Z$ for each $i \in \omega$. Let $\varphi$ be a Baire mapping $\varphi: X \mapsto Z$ from a space $X$ on a separable metric space $Z$. Then $B(\varphi(X))=$ $B(Z)$ is a dense subset of $B(X)$ and, hence, $B(Z)$ has property $S_{\text {fin }}(\mathcal{D}, \mathcal{D})$. Since $C_{p}(Z)$ is a dense subset of $B(Z), C_{p}(Z)$ also has property $S_{\text {fin }}(\mathcal{D}, \mathcal{D})$.

By Theorem 21 in [3] and [6], the space $Z$ has property $S_{f i n}(\Omega, \Omega)$. It follows that there is a sequence $\left\{F_{i}=\left\{W_{i}^{j_{1}}, \ldots, W_{i}^{j_{s}}\right\}\right\}_{i \in \omega}$ such that $F_{i} \subset \mathcal{B}_{i}$ and $\bigcup_{i \in \omega} F_{i} \in \Omega$. It follows that $\bigcup_{i \in \omega} F_{i}$ is Baire $\omega$-cover of $X$.

$(2) \Rightarrow(1)$. Assume that $X$ has property $S_{f i n}\left(B_{\Omega}, B_{\Omega}\right)$. Let $\left\{D_{i}\right\}_{i \in \omega}$ be a sequence countable dense subsets of $B(X)$. We claim that for any $f \in B(X)$ there is a sequence $\left\{F_{k}\right\}$ of finite subsets of $B(X)$ such that $F_{k} \subset D_{k}$ for each $k \in \omega$ and $f \in \overline{\bigcup\left\{F_{k}: k \in \omega\right\}}$.

Without loss of generality we can assume $f=\mathbf{0}$. Denote $W_{i}^{k}=\{x \in X$ : $\left.-\frac{1}{k}<f_{i}^{k}(x)<\frac{1}{k}\right\}$ for each $f_{i}^{k} \in D_{k}=\left\{f_{i}^{k}: i \in \omega\right\}$ and $k \in \omega$. 
If for each $j \in \omega$ there is $k(j)$ such that $W_{i(j)}^{k(j)}=X$, then a sequence $f_{k(j)}=f_{i(j)}^{k(j)}$ uniform convergence to $f$ and, hence, $f \in \overline{\left\{f_{k(j)}: j \in \omega\right\}}$.

We can assume that $W_{i}^{k} \neq X$ for any $k, i \in \omega$.

(a). $\left\{W_{i}^{k}\right\}_{i \in \omega}$ a sequence of Baire sets of $X$.

(b). For each $k \in \omega,\left\{W_{i}^{k}\right\}_{i \in \omega}$ is a $\omega$-cover of $X$.

By (2), $X$ has property $S_{\text {fin }}\left(\mathcal{B}_{\Omega}, \mathcal{B}_{\Omega}\right)$, hence, there is a sequence $\left\{S_{k}=\right.$ $\left.\left\{W_{i(1)}^{k}, \ldots, W_{i(s(k))}^{k}\right\}\right\}_{k \in \omega}$ such that $S_{k} \subset\left\{W_{i}^{k}\right\}_{i \in \omega}$ for each $k \in \omega$ and $\bigcup_{k \in \omega} S_{k}$ is a $\omega$-cover of $X$.

Consider $\left\{f_{i(j)}^{k}: j=1, \ldots, s(k), k \in \omega\right\}$ and we claim that

$f \in \overline{\left\{f_{i(j)}^{k}: j=1, \ldots, s(k), k \in \omega\right\}}$.

Let $K$ be a finite subset of $X, \epsilon>0$ and $U=<f, K, \epsilon>$ be a base neighborhood of $f$, then there is $k_{0} \in \omega$ such that $\frac{1}{k_{0}}<\epsilon$ and $K \subset W_{i\left(j^{\prime}\right)}^{k_{0}}$. It follows that $f_{i\left(j^{\prime}\right)}^{k_{0}} \in U$ for some $j^{\prime} \in\left\{1, \ldots, s\left(k_{0}\right)\right\}$.

Let $D=\left\{d_{n}: n \in \omega\right\}$ be a dense subspace of $B(X)$. Given a sequence $\left\{D_{i}\right\}_{i \in \omega}$ of dense subspace of $B(X)$, enumerate it as $\left\{D_{n, m}: n, m \in \omega\right\}$. For each $n \in \omega$, pick finite set $F_{n, m} \subset D_{n, m}$ so that $d_{n} \in \overline{\bigcup_{m \in \omega} F_{n, m}}$. Then $\bigcup_{m, n \in \omega} F_{n, m}$ is dense in $B(X)$.

$(2) \Leftrightarrow(3)$. By Theorem 4.1 and Theorem 2.2.

$(1) \Leftrightarrow(4)$. By Proposition 15 in [3] for countable dense subsets of $B(X)$.

\section{5. $B(X) \models S_{1}(\mathcal{D}, \mathcal{S})$}

Theorem 5.1. For a Tychonoff space $X$, the following statements are equivalent:

1. $B(X) \models S_{1}\left(\Omega_{x}, \Gamma_{x}\right)$;

2. $X \models S_{1}\left(\mathcal{B}_{\Omega}, \mathcal{B}_{\Gamma}\right)$.

Proof. (1) $\Rightarrow(2)$. Let $\left\{\mathcal{U}_{n}: n \in \omega\right\}$ be a sequence of Baire $\omega$-covers of $X$. We set $A_{n}=\left\{f \in B(X): f\left\lceil(X \backslash U)=0\right.\right.$ for some $\left.U \in \mathcal{U}_{n}\right\}$. It is not difficult to see that each $A_{n}$ is dense in $B(X)$ since each $\mathcal{U}_{n}$ is an $\omega$-cover of $X$ and $X$ is Tychonoff. Let $f$ be the constant function to 1 . By the assumption there exist $f_{n} \in A_{n}$ such that $\left\{f_{n}\right\}_{n \in \omega}$ converge to $f$.

For each $f_{n}$ we take $U_{n} \in \mathcal{U}_{n}$ such that $f_{n} \uparrow\left(X \backslash U_{n}\right)=0$.

Set $\mathcal{U}=\left\{U_{n}: n \in \omega\right\}$. For each finite subset $\left\{x_{1}, \ldots, x_{k}\right\}$ of $X$ we consider the basic open neighborhood of $f\left[x_{1}, \ldots, x_{k} ; W, \ldots, W\right]$, where $W=(0,2)$. 
Note that there is $n^{\prime} \in \omega$ such that $\left[x_{1}, \ldots, x_{k} ; W, \ldots, W\right]$ contains $f_{n}$ for $n>n^{\prime}$. This means $\left\{x_{1}, \ldots, x_{k}\right\} \subset U_{n}$ for $n>n^{\prime}$. Consequently $\mathcal{U}$ is an $\gamma$-cover of $X$.

$(2) \Rightarrow(1)$. Let $f \in \bigcap_{n} \overline{A_{n}}$, where $A_{n}$ is a subset of $B(X)$. Since $B(X)$ is homogeneous, we may think that $f$ is the constant function to the zero. We set $\mathcal{U}_{n}=\left\{g^{-1}(-1 / n, 1 / n): g \in A_{n}\right\}$ for each $n \in \omega$. For each $n \in \omega$ and each finite subset $\left\{x_{1}, \ldots, x_{k}\right\}$ of $X$ a neighborhood $\left[x_{1}, \ldots, x_{k} ; W, \ldots, W\right]$ of $f$, where $W=(-1 / n, 1 / n)$, contains some $g \in A_{n}$. This means that each $\mathcal{U}_{n}$ is Baire $\omega$-cover of $X$. In case the set $M=\left\{n \in \omega: X \in \mathcal{U}_{n}\right\}$ is infinite, choose $g_{m} \in A_{m} m \in M$ so that $g^{-1}(-1 / m, 1 / m)=X$, then $g_{m} \mapsto f$. So we may assume that there exists $n \in \omega$ such that for each $m \geq n$ and $g \in A_{m}$ $g^{-1}(-1 / m, 1 / m)$ is not $X$. For the sequence $\left\{\mathcal{U}_{m}: m \geq n\right\}$ of Baire $\omega$-covers there exist $f_{m} \in A_{m}$ such that

$\mathcal{U}=\left\{f_{m}^{-1}(-1 / m, 1 / m): m>n\right\}$ is a $\gamma$-cover of $X$. Let $\left[x_{1}, \ldots, x_{k} ; W, \ldots, W\right]$ be any basic open neighborhood of $f$, where $W=(-\epsilon, \epsilon), \epsilon>0$. There exists $m^{\prime} \geq n$ such that $\left\{x_{1}, \ldots, x_{k}\right\} \subset f_{m}^{-1}(-1 / m, 1 / m)$ and $1 / m<\epsilon$ for each $m>m^{\prime}$. This means $\left\{f_{m}\right\}$ converge to $f$.

Theorem 5.2. For a Tychonoff space $X$, the following statements are equivalent:

1. $B(X) \models S_{1}(\mathcal{D}, \mathcal{S})$ and it is separable;

2. $B(X)$ is strongly sequentially separable;

3. $X \models S_{1}\left(\mathcal{B}_{\Omega}, \mathcal{B}_{\Gamma}\right)$ and $i w(X)=\aleph_{0}$;

4. $B(X) \models S_{1}\left(\Omega_{x}, \Gamma_{x}\right)$ and it is separable;

5. $B(X) \models S_{1}\left(\mathcal{D}, \Gamma_{x}\right)$ and it is separable.

Proof. (1) $\Rightarrow(2)$. Let $D=\left\{d_{i}: i \in \omega\right\}$ be a countable dense subset of $B(X)$. By $S_{1}(\mathcal{D}, \mathcal{S})$, for sequence $\left\{D_{i}: D_{i}=D\right.$ and $\left.i \in \omega\right\}$ there is a set $\left\{d_{i}: i \in \omega\right\}$ such that for each $i, d_{i} \in D_{i}$, and $\left\{d_{i}: i \in \omega\right\}$ is a countable sequentially dense subset of $B(X)$. It follows that $D$ is a countable sequentially dense subset of $B(X)$.

$(2) \Leftrightarrow(3)$. By Theorem 3.4 in [10].

$(3) \Leftrightarrow(4)$. By Theorem 5.1.

$(4) \Rightarrow(5)$ is immediate.

$(5) \Rightarrow(2)$. Let $D \in \mathcal{D}, f \in B(X)$ and $\left\{D_{n}\right\}_{n \in \omega}$ such that $D_{n}=D$ for each $n \in \omega$. By (5), there is a set $\left\{f_{n}: n \in \omega\right\}$ such that for each $n, f_{n} \in D_{n}$, 
and $\left\{f_{n}\right\}_{n \in \omega}$ converge to $f$. It follows that $D$ is a sequentially dense subset of $B(X)$.

$(3) \Rightarrow(1)$. Let $\left\{D_{i}\right\}_{i \in \omega}$ be a sequence of countable dense subsets of $B(X)$. Since $X \models S_{1}\left(\mathcal{B}_{\Omega}, \mathcal{B}_{\Gamma}\right)$, then, $X \models S_{1}\left(\mathcal{B}_{\Omega}, \mathcal{B}_{\Omega}\right)$ and, by Theorem [3.2, $B(X)$ $\models S_{1}(\mathcal{D}, \mathcal{D})$. Then there is a sequence $\left\{d_{i}\right\}_{i \in \omega}$ such that for each $i, d_{i} \in D_{i}$, and $\left\{d_{i}: i \in \omega\right\}$ is a countable dense subset of $B(X)$. By (2), $\left\{d_{i}: i \in \omega\right\}$ is a countable sequentially dense subset of $B(X)$, i.e. $\left\{d_{i}: i \in \omega\right\} \in \mathcal{S}$.

Note that $S_{1}\left(\mathcal{B}_{\Omega}, \mathcal{B}_{\Gamma}\right)=S_{\text {fin }}\left(\mathcal{B}_{\Omega}, \mathcal{B}_{\Gamma}\right)($ see $[15])$.

Theorem 5.3. For a Tychonoff space $X$, the following statements are equivalent:

1. $B(X) \models S_{\text {fin }}\left(\Omega_{x}, \Gamma_{x}\right)$;

2. $X \models S_{\text {fin }}\left(\mathcal{B}_{\Omega}, \mathcal{B}_{\Gamma}\right)$.

Proof. By Theorem 5.1, it suffices to prove $(1) \Rightarrow(2)$.

$(1) \Rightarrow(2)$. Let $\left\{\mathcal{U}_{n}\right\}_{n \in \omega}$ be a sequence of Baire $\omega$-covers of $X$. We set $A_{n}=\left\{f \in B(X): f \uparrow(X \backslash U)=0\right.$ for some $\left.U \in \mathcal{U}_{n}\right\}$. It is not difficult to see that each $A_{n}$ is dense in $B(X)$ since each $\mathcal{U}_{n}$ is an $\omega$-cover of $X$ and $X$ is Tychonoff. Let $f$ be the constant function to 1 . By the assumption there exist $\left\{f_{n}^{i}\right\}_{i=1}^{k(n)} \subset A_{n}$ such that $\bigcup_{n \in \omega}\left\{f_{n}^{i}\right\}_{i=1}^{k(n)}$ converge to $f$. Note that a subsequence $\left\{f_{n}^{1}\right\}_{n \in \omega}$ also converge to $f$.

For each $f_{n}^{1}$ we take $U_{n} \in \mathcal{U}_{n}$ such that $f_{n}^{1} \uparrow\left(X \backslash U_{n}\right)=0$.

Set $\mathcal{U}=\left\{U_{n}: n \in \omega\right\}$. For each finite subset $\left\{x_{1}, \ldots, x_{k}\right\}$ of $X$ we consider the basic open neighborhood of $f\left[x_{1}, \ldots, x_{k} ; W, \ldots, W\right]$, where $W=(0,2)$.

Note that there is $n^{\prime} \in \omega$ such that $\left[x_{1}, \ldots, x_{k} ; W, \ldots, W\right]$ contains $f_{n}^{1}$ for $n>n^{\prime}$. This means $\left\{x_{1}, \ldots, x_{k}\right\} \subset U_{n}$ for $n>n^{\prime}$. Consequently $\mathcal{U}$ is an $\gamma$-cover of $X$.

Theorem 5.4. For a Tychonoff space $X$, the following statements are equivalent:

1. $B(X) \models S_{\text {fin }}(\mathcal{D}, \mathcal{S})$ and it is separable;

2. $B(X)$ is strongly sequentially separable;

3. $X \models S_{\text {fin }}\left(\mathcal{B}_{\Omega}, \mathcal{B}_{\Gamma}\right)$ and $i w(X)=\aleph_{0}$;

4. $B(X) \models S_{f i n}\left(\Omega_{x}, \Gamma_{x}\right)$ and it is separable;

5. $B(X) \models S_{\text {fin }}\left(\mathcal{D}, \Gamma_{x}\right)$ and it is separable.

Proof. By Theorem 5.3 and Theorem 5.2. 


\section{6. $B(X) \models S_{1}(\mathcal{S}, \mathcal{D})$}

Recall that $U_{f i n}(\mathcal{S}, \mathcal{D})$ is the selection hypothesis: whenever $\mathcal{U}_{1}, \mathcal{U}_{2}, \ldots \in$ $\mathcal{S}$, there are finite sets $\mathcal{F}_{n} \subseteq \mathcal{U}_{n}, n \in \omega$, such that $\left\{\bigcup \mathcal{F}_{n}: n \in \omega\right\} \in \mathcal{D}$. For a function space $B(X)$, we can represent the condition $\left\{\bigcup \mathcal{F}_{n}: n \in \omega\right\} \in \mathcal{D}$ as $\forall f \in B(X) \forall$ a base neighborhood $O(f)=<f, K, \epsilon>$ of $f$ where $\epsilon>0$ and $K=\left\{x_{1}, \ldots, x_{k}\right\}$ is a finite subset of $X$, there is $n^{\prime} \in \omega$ such that for each $j \in\{1, \ldots, k\}$ there is $g \in \mathcal{F}_{n^{\prime}}$ such that $g\left(x_{j}\right) \in\left(f\left(x_{j}\right)-\epsilon, f\left(x_{j}\right)+\epsilon\right)$.

Similarly, $U_{\text {fin }}\left(\Gamma_{0}, \Omega_{0}\right)$ : whenever $\mathcal{S}_{1}, \mathcal{S}_{2}, \ldots \in \Gamma_{0}$, there are finite sets $\mathcal{F}_{n} \subseteq \mathcal{S}_{n}, n \in \omega$, such that $\left\{\bigcup \mathcal{F}_{n}: n \in \omega\right\} \in \Omega_{0}$, i.e. for a base neighborhood $O(f)=<f, K, \epsilon>$ of $f=\mathbf{0}$ where $\epsilon>0$ and $K=\left\{x_{1}, \ldots, x_{k}\right\}$ is a finite subset of $X$, there is $n^{\prime} \in \omega$ such that for each $j \in\{1, \ldots, k\}$ there is $g \in \mathcal{F}_{n^{\prime}}$ such that $g\left(x_{j}\right) \in\left(f\left(x_{j}\right)-\epsilon, f\left(x_{j}\right)+\epsilon\right)$.

Theorem 6.1. For a Tychonoff space $X$, the following statements are equivalent:

1. $B(X) \models S_{1}\left(\Gamma_{x}, \Omega_{x}\right)$;

2. $X \models S_{1}\left(\mathcal{B}_{\Gamma}, \mathcal{B}_{\Omega}\right)$.

3. $B(X) \models S_{\text {fin }}\left(\Gamma_{x}, \Omega_{x}\right)$;

4. $B(X) \models U_{\text {fin }}\left(\Gamma_{x}, \Omega_{x}\right)$;

Proof. (1) $\Rightarrow(2)$. Let $B(X) \models S_{1}\left(\Gamma_{x}, \Omega_{x}\right)$ and $\left\{\mathcal{F}_{i}: i \in \omega\right\} \subset \mathcal{B}_{\Gamma}$. Let $\mathcal{F}_{i}=\left\{F_{i, k}: k \in \omega\right\}$ for each $i \in \omega$ and $f=\mathbf{0}$. For each $i \in \omega$ we consider a sequence $\left\{f_{i, k}\right\}_{k \in \omega}$ such that $f_{i, k} \uparrow F_{i, k}=0$ and $f_{i, k} \uparrow\left(X \backslash F_{i, k}\right)=1$ for each $k \in \omega$.

Since $\mathcal{F}_{i}$ is a Baire $\gamma$-cover of $X$, we have that $\left\{f_{i, k}\right\}_{k \in \omega}$ converge to $f$ for each $i \in \omega$.

Since $B(X) \models S_{1}\left(\Gamma_{x}, \Omega_{x}\right)$, there is a sequence $\left\{f_{i, k(i)}\right\}_{i \in \omega}$ such that for each $i, f_{i, k(i)} \in\left\{f_{i, k}: k \in \omega\right\}$, and $\left\{f_{i, k(i)}: i \in \omega\right\}$ is an element of $\Omega_{f}$.

Consider a sequence $\left\{F_{i, k(i)}: i \in \omega\right\}$.

(a). $F_{i, k(i)} \in \mathcal{F}_{i}$.

(b). $\left\{F_{i, k(i)}: i \in \omega\right\}$ is a $\omega$-cover of $X$.

Let $K$ be a finite subset of $X$ and $U=<f, K, \frac{1}{2}>$ be a base neighborhood of $f$, then there is $f_{i_{j_{0}}, k\left(i_{j_{0}}\right)} \in U$. It follows that $K \subset F_{i_{j_{0}}, k\left(i_{j_{0}}\right)}$. We thus get $X \models S_{1}\left(B_{\Gamma}, B_{\Omega}\right)$.

$(2) \Rightarrow(1)$. Let $\left\{f_{k, i}\right\}_{k \in \omega}$ be a sequence converge to $f$ for each $i \in \omega$. Without loss of generality we can assume that $f=\mathbf{0}$ and a set $W_{k}^{i}=\{x \in$ $\left.X:-\frac{1}{i}<f_{k, i}(x)<\frac{1}{i}\right\} \neq X$ for any $i \in \omega$. 
Consider $\mathcal{V}_{i}=\left\{W_{k}^{i}: k \in \omega\right\}$ for each $i \in \omega$.

(a). $\mathcal{V}_{i}$ is Baire cover of $X$.

(b). $\mathcal{V}_{i}$ is $\gamma$-cover of $X$. Since $\left\{f_{k, i}\right\}_{k \in \omega}$ converge to $f$, for each finite set $K \subset X$ there is $k_{0} \in \omega$ such that $f_{k, i} \in<f, K, \frac{1}{i}>$ for $k>k_{0}$. It follows that $K \subset W_{k}^{i}$ for any $k>k_{0}$.

By $X \models S_{1}\left(\mathcal{B}_{\Gamma}, \mathcal{B}_{\Omega}\right)$, there is a sequence $\left\{W_{k(i)}^{i}\right\}_{i \in \omega}$ such that for each $i$, $W_{k(i)}^{i} \in \mathcal{V}_{i}$, and $\left\{W_{k(i)}^{i}: i \in \omega\right\}$ is an element of $\mathcal{B}_{\Omega}$.

We claim that $f \in \overline{\left\{f_{k(i), i}: i \in \omega\right\}}$. Let $U=<f, K, \epsilon>$ be a base neighborhood of $f$ where $\epsilon>0$ and $K$ is a finite subset of $X$, then there are $i_{0}, i_{1} \in \omega$ such that $\frac{1}{i_{0}}<\epsilon, i_{1}>i_{0}$ and $W_{k\left(i_{1}\right)}^{i_{1}} \supset K$. It follows that $f_{k\left(i_{1}\right), i_{1}} \in<f, K, \epsilon>$ and, hence, $f \in \overline{\left\{f_{k(i), i}: i \in \omega\right\}}$.

$(1) \Rightarrow(3) \Rightarrow(4)$ is immediate.

(4) $\Rightarrow(2)$. Let $\left\{\mathcal{U}_{i}: i \in \omega\right\} \subset \mathcal{B}_{\Gamma}$. Let $\mathcal{U}_{i}=\left\{U_{i}^{m}: m \in \omega\right\}$ we consider $\mathcal{K}_{i}=\left\{f_{i}^{m} \in B(X): f_{i}^{m}\left\lceil U_{i}^{m}=0\right.\right.$ and $f_{i}^{m}\left\lceil\left(X \backslash U_{i}^{m}\right)=1\right.$ for $\left.m \in \omega\right\}$. Since $\mathcal{U}_{i}$ is a $\gamma$-cover of $X$, we have that $\mathcal{K}_{i}$ converge to $\mathbf{0}$ for each $i \in \omega$. By $B(X)$ $\models U_{f i n}\left(\Gamma_{x}, \Omega_{x}\right)$, there are finite sets $F_{i}=\left\{f_{i}^{m_{1}}, \ldots, f_{i}^{m_{s(i)}}\right\} \subseteq \mathcal{K}_{i}$ such that $\left\{\bigcup F_{i}: i \in \omega\right\} \in \Omega_{0}$. Note that $\left\{\bigcup\left\{U_{i}^{m_{1}}, \ldots, U_{i}^{m_{s(i)}}\right\}: i \in \omega\right\} \in B_{\Omega}$. It follows that $X \models U_{\text {fin }}\left(\mathcal{B}_{\Gamma}, \mathcal{B}_{\Omega}\right)$. By Theorem 9 in [15], $S_{1}\left(\mathcal{B}_{\Gamma}, \mathcal{B}_{\Omega}\right) \Leftrightarrow S_{\text {fin }}\left(\mathcal{B}_{\Gamma}, \mathcal{B}_{\Omega}\right) \Leftrightarrow$ $U_{\text {fin }}\left(\mathcal{B}_{\Gamma}, \mathcal{B}_{\Omega}\right)$.

Theorem 6.2. For a Tychonoff space $X$, the following statements are equivalent:

1. $B(X) \models S_{1}(\mathcal{S}, \mathcal{D})$ and it is sequentially separable;

2. $X \models S_{1}\left(\mathcal{B}_{\Gamma}, \mathcal{B}_{\Omega}\right)$ and $X \models O$;

3. $B(X) \models S_{1}\left(\Gamma_{x}, \Omega_{x}\right)$ and it is sequentially separable;

4. $B(X) \models S_{1}\left(\mathcal{S}, \Omega_{x}\right)$ and it is sequentially separable;

5. $B(X) \models S_{\text {fin }}(\mathcal{S}, \mathcal{D})$ and it is sequentially separable;

6. $B(X) \models U_{\text {fin }}(\mathcal{S}, \mathcal{D})$ and it is sequentially separable;

7. $B(X) \models S_{\text {fin }}\left(\mathcal{S}, \Omega_{x}\right)$ and it is sequentially separable;

8. $B(X) \models U_{\text {fin }}\left(\mathcal{S}, \Omega_{x}\right)$ and it is sequentially separable.

Proof. (1) $\Rightarrow(5) \Rightarrow(6)$ is immediate.

$(6) \Rightarrow(2)$. Let $\left\{\mathcal{F}_{i}: i \in \omega\right\} \subset \mathcal{B}_{\Gamma}$ where $\mathcal{F}_{i}=\left\{F_{i}^{m}: m \in \omega\right\}$ and $\mathcal{S}=\left\{h_{m}: m \in \omega\right\}$ be a countable sequentially dense subset of $B(X)$. For each $i \in \omega$ we consider a countable sequentially dense subset $\mathcal{S}_{i}$ of $B(X)$. 
where $\mathcal{S}_{i}:=\left\{f_{i}^{m} \in B(X): f_{i}^{m} \uparrow F_{i}^{m}=h_{m}\right.$ and $f_{i}^{m} \uparrow\left(X \backslash F_{i}^{m}\right)=1$ for $m \in \omega\}$.

Since $\mathcal{F}_{i}$ is a Baire $\gamma$-cover of $X$ and $\mathcal{S}$ is a countable sequentially dense subset of $B(X)$, we have that $\mathcal{S}_{i}$ is a countable sequentially dense subset of $B(X)$ for each $i \in \omega$. Let $h \in B(X)$, there is a sequence $\left\{h_{m_{s}}\right\}_{s \in \omega}$ such that $\left\{h_{m_{s}}: s \in \omega\right\} \subset \mathcal{S}$ and $\left\{h_{m_{s}}\right\}_{s \in \omega}$ converge to $h$. Let $K$ be a finite subset of $X, \epsilon>0$ and $W=<h, K, \epsilon>$ be a base neighborhood of $h$, then there is a number $m_{0}$ such that $K \subset F_{i}^{m}$ for $m>m_{0}$ and $h_{m_{s}} \in W$ for $m_{s}>m_{0}$. Since $f_{i}^{m_{s}}\left\lceil K=h_{m_{s}} \uparrow K\right.$ for each $m_{s}>m_{0}, f_{i}^{m_{s}} \in W$ for each $m_{s}>m_{0}$. It follows that a sequence $\left\{f_{i}^{m_{s}}\right\}_{s \in \omega}$ converge to $h$.

By $B(X) \models U_{f i n}(\mathcal{S}, \mathcal{D})$, there are finite sets $F_{i}=\left\{f_{i}^{m_{1}}, \ldots, f_{i}^{m_{s(i)}}\right\} \subseteq \mathcal{S}_{i}$ such that $\left\{\bigcup F_{i}: i \in \omega\right\} \in \mathcal{D}$.

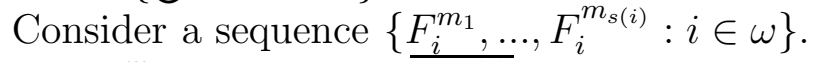

(a). $F_{i}^{m_{k}} \in \mathcal{F}_{i}$ for $k \in \overline{1, s(i)}$.

(b). $\left\{\bigcup_{k=1}^{s(i)} F_{i}^{m_{k}}: i \in \omega\right\}$ is a $\omega$-cover of $X$.

Let $K$ be a finite subset of $X$ and $U=<\mathbf{0}, K, \frac{1}{2}>$ be a base neighborhood

of $\mathbf{0}$, then there is $F_{i}$ such that $K \subset \bigcup_{k=1}^{s(i)} F_{i}^{m_{k}}$. We thus get $X \models U_{\text {fin }}\left(B_{\Gamma}, B_{\Omega}\right)$. By Theorem 9 in [15], $S_{1}\left(\mathcal{B}_{\Gamma}, \mathcal{B}_{\Omega}\right) \Leftrightarrow S_{\text {fin }}\left(\mathcal{B}_{\Gamma}, \mathcal{B}_{\Omega}\right) \Leftrightarrow U_{\text {fin }}\left(\mathcal{B}_{\Gamma}, \mathcal{B}_{\Omega}\right)$.

$(2) \Rightarrow(3)$. Let $X \models S_{1}\left(B_{\Gamma}, B_{\Omega}\right)$ and $\left\{f_{i, m}\right\}_{m \in \omega}$ converge to $\mathbf{0}$ for each $i \in \omega$.

Consider $\mathcal{F}_{i}=\left\{F_{i, m}: m \in \omega\right\}=\left\{f_{i, m}^{-1}\left(-\frac{1}{i}, \frac{1}{i}\right): m \in \omega\right\}$ for each $i \in \omega$. Without loss of generality we can assume that a set $F_{i, m} \neq X$ for any $i, m \in$ $\omega$. Otherwise there is sequence $\left\{f_{i_{k}, m_{k}}\right\}_{k \in \omega}$ such that $\left\{f_{i_{k}, m_{k}}\right\}_{k \in \omega}$ uniform converge to $\mathbf{0}$ and $\left\{f_{i_{k}, m_{k}}: k \in \omega\right\} \in \Omega_{\mathbf{0}}$.

Note that $\mathcal{F}_{i}$ is Baire $\gamma$-cover of $X$ for each $i \in \omega$. By $X \models S_{1}\left(\mathcal{B}_{\Gamma}, \mathcal{B}_{\Omega}\right)$, there is a sequence $\left\{F_{i, m(i)}: i \in \omega\right\}$ such that for each $i, F_{i, m(i)} \in \mathcal{F}_{i}$, and $\left\{F_{i, m(i)}: i \in \omega\right\}$ is an element of $\mathcal{B}_{\Omega}$.

We claim that $\mathbf{0} \in \overline{\left\{f_{i, m(i)}: i \in \omega\right\}}$. Let $W=<\mathbf{0}, K, \epsilon>$ be a base neighborhood of $\mathbf{0}$ where $\epsilon>0$ and $K$ is a finite subset of $X$, then there are $i_{0}, i_{1} \in \omega$ such that $\frac{1}{i_{0}}<\epsilon, i_{1}>i_{0}$ and $F_{i_{1}, m\left(i_{1}\right)} \supset K$. It follows that $f_{i_{1}, m\left(i_{1}\right)} \in<\mathbf{0}, K, \epsilon>$ and, hence, $\mathbf{0} \in \overline{\left\{f_{i, m(i)}: i \in \omega\right\}}$ and $B(X) \models$ $S_{1}\left(\Gamma_{x}, \Omega_{x}\right)$

$(3) \Rightarrow(4)$ is immediate.

$(4) \Rightarrow(1)$. Suppose that $B(X)$ is a sequentially separable and $B(X) \models$ $S_{1}\left(\mathcal{S}, \Omega_{x}\right)$. Let $D=\left\{d_{n}: n \in \omega\right\}$ be a dense subspace of $B(X)$. Given a 
sequence of sequentially dense subspaces of $B(X)$, enumerate it as $\left\{S_{n, m}\right.$ : $n, m \in \omega\}$. For each $n \in \omega$, pick $d_{n, m} \in S_{n, m}$ so that $d_{n} \in \overline{\left\{d_{n, m}: m \in \omega\right\}}$. Then $\left\{d_{n, m}: m, n \in \omega\right\}$ is dense in $B(X)$.

$(4) \Rightarrow(7) \Rightarrow(8)$ is immediate.

$(8) \Rightarrow(6)$. Similarly to the implication $(4) \Rightarrow(1)$.

\section{7. $B(X) \models S_{1}(\mathcal{S}, \mathcal{S})$}

Recall that $U_{\text {fin }}(\mathcal{S}, \mathcal{S})$ is the selection hypothesis: whenever $\mathcal{U}_{1}, \mathcal{U}_{2}, \ldots \in$ $\mathcal{S}$, there are finite sets $\mathcal{F}_{n} \subseteq \mathcal{U}_{n}, n \in \omega$, such that $\left\{\bigcup \mathcal{F}_{n}: n \in \omega\right\} \in \mathcal{S}$. For a function space $B(X)$, we can represent the condition $\left\{\bigcup \mathcal{F}_{n}: n \in \omega\right\} \in \mathcal{S}$ as $\forall f \in B(X) \forall$ a base neighborhood $O(f)=<f, K, \epsilon>$ of $f$ where $\epsilon>0$ and $K=\left\{x_{1}, \ldots, x_{k}\right\}$ is a finite subset of $X$, there is $n^{\prime} \in \omega$ such that for each $n>$ $n^{\prime}$ and $j \in\{1, \ldots, k\}$ there is $g \in \mathcal{F}_{n}$ such that $g\left(x_{j}\right) \in\left(f\left(x_{j}\right)-\epsilon, f\left(x_{j}\right)+\epsilon\right)$.

Similarly, $U_{f i n}\left(\Gamma_{0}, \Gamma_{0}\right)$ : whenever $\mathcal{S}_{1}, \mathcal{S}_{2}, \ldots \in \Gamma_{0}$, there are finite sets $\mathcal{F}_{n} \subseteq \mathcal{S}_{n}, n \in \omega$, such that $\left\{\bigcup \mathcal{F}_{n}: n \in \omega\right\} \in \Gamma_{0}$, i.e. for a base neighborhood $O(f)=<f, K, \epsilon>$ of $f=\mathbf{0}$ where $\epsilon>0$ and $K=\left\{x_{1}, \ldots, x_{k}\right\}$ is a finite subset of $X$, there is $n^{\prime} \in \omega$ such that for each $n>n^{\prime}$ and $j \in\{1, \ldots, k\}$ there is $g \in \mathcal{F}_{n}$ such that $g\left(x_{j}\right) \in\left(f\left(x_{j}\right)-\epsilon, f\left(x_{j}\right)+\epsilon\right)$.

Theorem 7.1. For a Tychonoff space $X$, the following statements are equivalent:

1. $B(X) \models S_{1}\left(\Gamma_{x}, \Gamma_{x}\right)$;

2. $X \models S_{1}\left(\mathcal{B}_{\Gamma}, \mathcal{B}_{\Gamma}\right)$;

3. $B(X) \models S_{\text {fin }}\left(\Gamma_{x}, \Gamma_{x}\right)$;

4. $B(X) \models U_{\text {fin }}\left(\Gamma_{x}, \Gamma_{x}\right)$.

Proof. (1) $\Rightarrow(3) \Rightarrow(4)$ is immediate.

$(4) \Rightarrow(2)$. Let $\left\{\mathcal{U}_{i}: i \in \omega\right\} \subset \mathcal{B}_{\Gamma}$. For each $i \in \omega$ we consider a subset $\mathcal{S}_{i}$ of $B(X)$ and $\mathcal{U}_{i}=\left\{U_{i}^{m}: m \in \omega\right\}$ where

$\mathcal{S}_{i}:=\left\{f_{i}^{m} \in B(X): f_{i}^{m} \uparrow U_{i}^{m}=0\right.$ and $f_{i}^{m} \uparrow\left(X \backslash U_{i}^{m}\right)=1$ for $\left.m \in \omega\right\}$.

Since $\mathcal{U}_{i}$ is a $\gamma$-cover of $X$, we have that $\mathcal{S}_{i}$ converge to $\mathbf{0}$, i.e. $\mathcal{S}_{i} \in \Gamma_{0}$ for each $i \in \omega$.

Since $B(X) \models U_{\text {fin }}\left(\Gamma_{x}, \Gamma_{x}\right)$, there is a sequence $\left\{\mathcal{F}_{i}\right\}_{i \in \omega}=\left\{f_{i}^{m_{1}}, \ldots, f_{i}^{m_{k(i)}}\right.$ : $i \in \omega\}$ such that for each $i, \mathcal{F}_{i} \subseteq \mathcal{S}_{i}$, and $\left\{\bigcup \mathcal{F}_{i}: i \in \omega\right\} \in \Gamma_{0}$.

Consider a sequence $\left\{W_{i}\right\}_{i \in \omega}=\left\{U_{i}^{m_{1}}, \ldots, U_{i}^{m_{k(i)}}: i \in \omega\right\}$.

(a). $W_{i} \subset \mathcal{U}_{i}$. 
(b). $\left\{\bigcup W_{i}: i \in \omega\right\}$ is a $\gamma$-cover of $X$.

Let $K=\left\{x_{1}, \ldots, x_{s}\right\}$ be a finite subset of $X$ and $U=<\mathbf{0}, K, \frac{1}{2}>$ be a base neighborhood of $\mathbf{0}$, then there exists $i_{0} \in \omega$ such that for each $i>i_{0}$ and

$j \in\{1, \ldots, s\}$ there is $g \in \mathcal{F}_{i}$ such that $g\left(x_{j}\right) \in\left(-\frac{1}{2}, \frac{1}{2}\right)$.

It follows that $K \subset \bigcup_{j=1}^{k(i)} U_{i}^{m_{j}}$ for $i>i_{0}$. We thus get $X \models U_{\text {fin }}\left(\mathcal{B}_{\Gamma}, \mathcal{B}_{\Gamma}\right)$.

By Theorem 1 in [15], $S_{1}\left(\mathcal{B}_{\Gamma}, \mathcal{B}_{\Gamma}\right) \Leftrightarrow S_{\text {fin }}\left(\mathcal{B}_{\Gamma}, \mathcal{B}_{\Gamma}\right) \Leftrightarrow U_{\text {fin }}\left(\mathcal{B}_{\Gamma}, \mathcal{B}_{\Gamma}\right)$.

$(2) \Rightarrow(1)$. For each $n \in \omega$, let $\left\{f_{n, m}\right\}_{m \in \omega}$ be a sequence of Baire functions on $X$ converging pointwise to 0 . For $n, m \in \omega$, let $U_{n, m}=\{x \in X$ : $\left.\left|f_{n, m}(x)\right|<\frac{1}{2^{n}}\right\}$.

For each $n \in \omega$, we put $\mathcal{U}_{n}=\left\{U_{n, m}: m \in \omega\right\}$. If the set $\{n \in \omega: X \in$ $\left.\mathcal{U}_{n}\right\}$ is infinite, $X=U_{n_{0}, m_{0}}=U_{n_{1}, m_{1}}=\ldots$ for some sequences $\left\{n_{j}\right\}_{j \in \omega}$ and $\left\{m_{j}\right\}_{j \in \omega}$, where $\left\{n_{j}\right\}_{j \in \omega}$ is strictly increasing. This means that $\left\{f_{n_{j}, m_{j}}\right\}_{j \in \omega}$ is a sequence converging uniformly to 0 . If the set $\left\{n \in \omega: X \in \mathcal{U}_{n}\right\}$ is finite, by removing such finitely many $n$ 's we assume $U_{n, m} \neq X$ for $n, m \in \omega$. Note that each $\mathcal{U}_{n}$ is a $\gamma$-cover of $X$. By $X \models S_{1}\left(B_{\Gamma}, B_{\Gamma}\right)$, there is a sequence $\left\{U_{n, m(n)}\right\}$ such that $U_{n, m(n)} \in \mathcal{U}_{n}$ for each $n \in \omega$ and $\left\{U_{n, m(n)}: n \in \omega\right\}$ is a $\gamma$-cover of $X$. Let $F$ be a finite subset of $X$ and let $\epsilon$ a positive real number. Because of $U_{n, m(n)} \neq X$ for $n \in \omega$, there is $n^{\prime} \in \omega$ such that $F \subset U_{n, m(n)}$ for each $n>n^{\prime}$ and $\frac{1}{2^{n^{\prime}}}<\epsilon$. Then $\left|f_{n, m(n)}(x)\right|<\epsilon$ for any $x \in F$ and $n>n^{\prime}$. Hence $\left\{f_{n, m(n)}\right\}$ converge to $\mathbf{0}$. Thus $B(X) \models S_{1}\left(\Gamma_{x}, \Gamma_{x}\right)$.

Theorem 7.2. For a Tychonoff space $X$, the following statements are equivalent:

1. $B(X) \models S_{1}(\mathcal{S}, \mathcal{S})$ and it is sequentially separable;

2. $X \models S_{1}\left(\mathcal{B}_{\Gamma}, \mathcal{B}_{\Gamma}\right)$ and $X \models O$;

3. $B(X) \models S_{1}\left(\Gamma_{x}, \Gamma_{x}\right)$ and it is sequentially separable;

4. $B(X) \models S_{1}\left(\mathcal{S}, \Gamma_{x}\right)$ and it is sequentially separable;

5. $B(X) \models S_{\text {fin }}(\mathcal{S}, \mathcal{S})$ and it is sequentially separable;

6. $B(X) \models U_{f i n}(\mathcal{S}, \mathcal{S})$ and it is sequentially separable;

7. $B(X) \models S_{f i n}\left(\mathcal{S}, \Gamma_{x}\right)$ and it is sequentially separable;

8. $B(X) \models U_{\text {fin }}\left(\mathcal{S}, \Gamma_{x}\right)$ and it is sequentially separable.

Proof. (1) $\Rightarrow(2)$. Let $\left\{\mathcal{F}_{i}: i \in \omega\right\} \subset \mathcal{B}_{\Gamma}$ where $\mathcal{F}_{i}=\left\{F_{i}^{m}: m \in \omega\right\}$ and $\mathcal{S}=\left\{h_{k}: k \in \omega\right\}$ be a countable sequentially dense subset of $B(X)$. For each 
$i \in \omega$ we consider a countable sequentially dense subset $\mathcal{S}_{i}$ of $B(X)$ where $\mathcal{S}_{i}:=\left\{f \in B(X): f \uparrow F_{i}^{m}=h_{m}\right.$ and $f \uparrow\left(X \backslash F_{i}^{m}\right)=1$ for $\left.m \in \omega\right\}$.

Since $\mathcal{F}_{i}$ is a Baire $\gamma$-cover of $X$ and $\mathcal{S}$ is a countable sequentially dense subset of $B(X)$, we have that $\mathcal{S}_{i}$ is a countable sequentially dense subset of $B(X)$ for each $i \in \omega$.

Let $h \in B(X)$, there is a sequence $\left\{h_{s}\right\}_{s \in \omega} \subset \mathcal{S}$ such that $\left\{h_{s}\right\}_{s \in \omega}$ converge to $h$. Let $K$ be a finite subset of $X$ and $W=<h, K, \epsilon>$ be a base neighborhood of $h$, then there is a number $m_{0}$ such that $K \subset F_{i}^{m}$ for $m>m_{0}$ and $h_{s} \in W$ for $s>m_{0}$. Since $f_{i}^{s} \uparrow K=h_{s} \uparrow K$ for each $s>m_{0}, f_{i}^{s} \in W$ for each $s>m_{0}$. It follows that a sequence $\left\{f_{i}^{s}\right\}_{s \in \omega}$ converge to $h$.

Since $B(X) \models S_{1}(\mathcal{S}, \mathcal{S})$, there is a set $\left\{f_{i}^{m(i)}: i \in \omega\right\}$ such that for each $i, f_{i}^{m(i)} \in \mathcal{S}_{i}$, and $\left\{f_{i}^{m(i)}: i \in \omega\right\}$ is an element of $\mathcal{S}$.

For $\mathbf{0} \in B(X)$ there is a set $\left\{f_{i_{j}}^{m(i)_{j}}\right\}_{j \in \omega} \subset\left\{f_{i}^{m(i)}: i \in \omega\right\}$ such that $\left\{f_{i_{j}}^{m(i)_{j}}\right\}_{j \in \omega}$ converge to $\mathbf{0}$. Consider a set $\left\{F_{i_{j}}^{m(i)_{j}}: j \in \omega\right\}$.

(1) $F_{i_{j}}^{m(i)_{j}} \in \mathcal{F}_{i_{j}}$.

(2) $\left\{F_{i_{j}}^{m(i)_{j}}: j \in \omega\right\}$ is a $\gamma$-cover of $X$.

Let $K$ be a finite subset of $X$ and $U=<\mathbf{0}, K, \frac{1}{2}>$ be a base neighborhood of $\mathbf{0}$, then there is a number $j_{0}$ such that $f_{i_{j}}^{m(i)_{j}} \in U$ for any $j>j_{0}$. It follows that $K \subset F_{i_{j}}^{m(i)_{j}}$ for any $j>j_{0}$. We thus get $X \in U_{\text {fin }}\left(\mathcal{B}_{\Gamma}, \mathcal{B}_{\Gamma}\right)$. Note that $U_{\text {fin }}\left(\mathcal{B}_{\Gamma}, \mathcal{B}_{\Gamma}\right)=S_{1}\left(\mathcal{B}_{\Gamma}, \mathcal{B}_{\Gamma}\right)=S_{\text {fin }}\left(\mathcal{B}_{\Gamma}, \mathcal{B}_{\Gamma}\right)$ (see Theorem 1 in [15]).

$(2) \Rightarrow(1)$. Let $\left\{S_{i}\right\} \subset \mathcal{S}$ and $S \in \mathcal{S}$. Consider a topology $\tau$ generated by the family $\mathcal{P}=\left\{f^{-1}(G): G\right.$ is an open set of $\mathbb{R}$ and $\left.f \in S \cup \bigcup_{i \in \omega} S_{i}\right\}$. A space $Y=(X, \tau)$ is a separable metrizable space because $P=S \cup \bigcup_{i \in \omega} S_{i}$ is a countable dense subset of $B(X)$. Note that a function $f \in P$, considered as map from $Y$ to $\mathbb{R}$, is a continuous function. Note also that the identity map $\varphi$ from $X$ on $Y$, is a Borel bijection. By Corollary 12 in [4], $Y$ is a $Q N$-space and, hence, by Corollary 20 in [16], $Y$ has a property $S_{1}\left(\mathcal{B}_{\Gamma}, \mathcal{B}_{\Gamma}\right)$. By Corollary 21 in [16], $B(Y)$ is an $\alpha_{2}$ space and, hence, $B(Y)$ has property $S_{1}\left(\Gamma_{z}, \Gamma_{z}\right)$ for each $z \in B(Y)$.

We claim that there is a sequence $B=\left\{b_{i}: i \in \omega\right\}$ such that for each $i, b_{i} \in S_{i} \subset C(Y)$, and, $S \subset[B]_{\text {seq }}$, i.e. for each $d \in S \subset C(Y)$ there is a sequence $\left\{b_{i_{k}}: k \in \omega\right\} \subset B$ such that $\left\{b_{i_{k}}: k \in \omega\right\}$ converges to $d$.

Let $q: \omega \mapsto \omega \times \omega$ be a bijection. Then we enumerate $\left\{S_{i}\right\}$ as $\left\{S_{q(i)}\right\}$. For each $d_{n} \in S$ there are sequences $s_{n, m} \subset S_{n, m}$ such that $s_{n, m}$ converges to $d_{n}$ for each $m \in \omega$. By property $S_{1}\left(\Gamma_{z}, \Gamma_{z}\right)$ of $B(Y)$, there is a sequence 
$\left\{b_{n, m}: m \in \omega\right\}$ such that for each $m, b_{n, m} \in s_{n, m}$, and, $\left\{b_{n, m}\right\}$ converges to $d_{n}$. Denote $B=\left\{b_{n, m}: n, m \in \omega\right\}$. By construction of $B$, we have that $S \subset[B]_{\text {seq }}$.

Note that $B_{1}(X)=B(X)$ and $\varphi(B(Y))=\varphi\left(B_{1}(Y)\right)=B(X)$.

We claim that $B \in \mathcal{S}$. Let $f \in B(Y)$ and $\left\{f_{k}: k \in \omega\right\} \subset S$ such that $\left\{f_{k}\right\}$ converge to $f$. For each $k \in \omega$ there is $\left\{f_{k}^{n}: n \in \omega\right\} \subset B$ such that $\left\{f_{k}^{n}\right\}$ converge to $f_{k}$. Since $Y$ is a $Q N$-space, by Theorem 16 in [4], there exists an unbounded $\beta \in \omega^{\omega}$ such that $\left\{f_{\beta(n)}^{n}\right\}$ converge to $f$ on $Y$. It follows that $\left\{f_{\beta(n)}^{n}\right\}$ converge to $f$ on $X$ and $[B]_{\text {seq }}=B(X)$.

$(2) \Leftrightarrow(3)$. By Theorem 7.1 and Theorem 2.4 .

$(3) \Rightarrow(4)$ is immediate.

$(4) \Rightarrow(2)$. Let $B(X) \models S_{1}\left(\mathcal{S}, \Gamma_{x}\right)$ and $B(X)$ be a sequentially separable. Let $\left\{\mathcal{U}_{i}\right\} \subset \mathcal{B}_{\Gamma}$ and $\mathcal{S}=\left\{h_{m}\right\}_{m \in \omega}$ be a countable sequentially dense subset of $B(X)$. For each $i \in \omega$ we consider a countable sequentially dense subset $\mathcal{S}_{i}$ of $B(X)$ and $\mathcal{U}_{i}=\left\{U_{i}^{m}\right\}_{m \in \omega}$ where

$\mathcal{S}_{i}=\left\{f_{i}^{m}\right\}:=\left\{f_{i}^{m} \in B(X): f_{i}^{m} \uparrow U_{i}^{m}=h_{m}\right.$ and $f_{i}^{m} \uparrow\left(X \backslash U_{i}^{m}\right)=1$ for $m \in \omega\}$.

Since $\mathcal{F}_{i}=\left\{U_{i}^{m}\right\}_{m \in \omega}$ is a $\gamma$-cover of $X$ and $\mathcal{S}$ is a countable sequentially dense subset of $B(X)$, we have that $\mathcal{S}_{i}$ is a countable sequentially dense subset of $B(X)$ for each $i \in \omega$.

Let $h \in B(X)$, there is a sequence $\left\{h_{m_{s}}\right\}_{s \in \omega} \subset \mathcal{S}$ such that $\left\{h_{m_{s}}\right\}_{s \in \omega}$ converge to $h$. Let $K$ be a finite subset of $X, \epsilon>0$ and $W=<h, K, \epsilon>$ be a base neighborhood of $h$, then there is a number $m_{0}$ such that $K \subset U_{i}^{m}$ for $m>m_{0}$ and $h_{m_{s}} \in W$ for $m_{s}>m_{0}$. Since $f_{i}^{m_{s}} \uparrow K=h_{m_{s}} \uparrow K$ for each $m_{s}>m_{0}, f_{i}^{m_{s}} \in W$ for each $m_{s}>m_{0}$. It follows that a sequence $\left\{f_{i}^{m_{s}}\right\}_{s \in \omega}$ converge to $h$.

By $B(X) \models S_{1}\left(\mathcal{S}, \Gamma_{x}\right)$, there is a sequence $\left\{f_{i}^{m(i)}: i \in \omega\right\}$ such that for each $i, f_{i}^{m(i)} \in \mathcal{S}_{i}$, and $\left\{f_{i}^{m(i)}: i \in \omega\right\}$ is an element of $\Gamma_{0}$.

Consider a sequence $\left\{U_{i}^{m(i)}: i \in \omega\right\}$.

(a). $U_{i}^{m(i)} \in \mathcal{U}_{i}$.

(b). $\left\{U_{i}^{m(i)}: i \in \omega\right\}$ is a $\gamma$-cover of $X$.

Let $K$ be a finite subset of $X$ and $U=<\mathbf{0}, K, \frac{1}{2}>$ be a base neighborhood of $\mathbf{0}$, then there is $j_{0} \in \omega$ such that $f_{i_{j}}^{m(i)_{j}} \in U$ for each $j>j_{0}$. It follows that $K \subset U_{i_{j}}^{m(i)_{j}}$ for each $j>j_{0}$. We thus get $X \models S_{1}\left(\mathcal{B}_{\Gamma}, \mathcal{B}_{\Gamma}\right)$. By Theorem 2.4, $X \models O$.

$(1) \Rightarrow(5) \Rightarrow(6)$ and $(4) \Rightarrow(7) \Rightarrow(8)$ are immediate. 
$(8) \Rightarrow(2) . \quad B(X) \models U_{\text {fin }}\left(\mathcal{S}, \Gamma_{x}\right) \Rightarrow X \models U_{\text {fin }}\left(\mathcal{B}_{\Gamma}, \mathcal{B}_{\Gamma}\right)$ is proved similarly to the implication $(4) \Rightarrow(2)$. By Theorem 1 in [15], $S_{1}\left(\mathcal{B}_{\Gamma}, \mathcal{B}_{\Gamma}\right) \Leftrightarrow$ $S_{\text {fin }}\left(\mathcal{B}_{\Gamma}, \mathcal{B}_{\Gamma}\right) \Leftrightarrow U_{\text {fin }}\left(\mathcal{B}_{\Gamma}, \mathcal{B}_{\Gamma}\right)$.

$(6) \Rightarrow(2)$. Similarly to the implication $(1) \Rightarrow(2)$.

\section{8. $B(X) \models S_{1}(\mathcal{A}, \mathcal{A})$}

Definition 8.1. A set $A \subseteq B(X)$ will be called $n$-dense in $B(X)$, if for each $n$-finite set $\left\{x_{1}, \ldots, x_{n}\right\} \subset X$ such that $x_{i} \neq x_{j}$ for $i \neq j$ and an open sets $W_{1}, \ldots, W_{n}$ in $\mathbb{R}$ there is $f \in A$ such that $f\left(x_{i}\right) \in W_{i}$ for $i \in \overline{1, n}$.

Obviously, that if $A$ is a $n$-dense set of $B(X)$ for each $n \in \omega$ then $A$ is a dense set of $B(X)$.

For a space $B(X)$ we denote:

$\mathcal{A}_{n}$ — the family of a countable $n$-dense subsets of $B(X)$.

If $n=1$, then we denote $\mathcal{A}$ instead of $\mathcal{A}_{1}$.

Definition 8.2. Let $f \in B(X)$. A set $B \subseteq B(X)$ will be called $n$-dense at point $f$, if for each $n$-finite set $\left\{x_{1}, \ldots, x_{n}\right\} \subset X$ and $\epsilon>0$ there is $h \in B$ such that $h\left(x_{i}\right) \in\left(f\left(x_{i}\right)-\epsilon, f\left(x_{i}\right)+\epsilon\right)$ for $i \in \overline{1, n}$.

Obviously, that if $B$ is a $n$-dense at point $f$ for each $n \in \omega$ then $f \in \bar{B}$.

For a space $B(X)$ we denote:

$\mathcal{B}_{n, f}$ — the family of a countable $n$-dense at point $f$ subsets of $B(X)$.

If $n=1$, then we denote $\mathcal{B}_{f}$ instead of $\mathcal{B}_{1, f}$.

Let $\mathcal{B}$ be a Baire cover of $X$ and $n \in \mathbb{N}$.

- $\mathcal{B}$ is an $n$-cover of $X$ if for each $F \subset X$ with $|F| \leq n$, there is $B \in \mathcal{B}$ such that $F \subset B$.

Theorem 8.3. For a space $X$, the following statements are equivalent:

1. $B(X) \models S_{1}(\mathcal{A}, \mathcal{A})$;

2. $X \models S_{1}(\mathcal{B}, \mathcal{B})$;

3. $B(X) \models S_{1}\left(\mathcal{B}_{f}, \mathcal{B}_{f}\right)$;

4. $B(X) \models S_{1}\left(\mathcal{A}, \mathcal{B}_{f}\right)$;

Proof. $(1) \Rightarrow(2)$. Let $\left\{\mathcal{B}_{n}: n \in \omega\right\}$ be a sequence of countable Baire covers of $X$. We set $A_{n}=\left\{f \in B(X): f \uparrow(X \backslash U)=1\right.$ and $f \uparrow U=q$ for $U \in \mathcal{B}_{n}$ 
and $q \in \mathbb{Q}\}$. It is not difficult to see that each $A_{n}$ is countable 1-dense subset of $B(X)$ since each $\mathcal{B}_{n}$ is a cover of $X$.

By the assumption there exist $f_{n} \in A_{n}$ such that $\left\{f_{n}: n \in \omega\right\} \in \mathcal{A}$.

For each $f_{n}$ we take $U_{n} \in \mathcal{B}_{n}$ such that $f_{n} \uparrow\left(X \backslash U_{n}\right)=1$.

Set $\mathcal{U}=\left\{U_{n}: n \in \omega\right\}$. For $x \in X$ we consider the basic open neighborhood of $\mathbf{0}[x, W]$, where $W=\left(-\frac{1}{2}, \frac{1}{2}\right)$.

Note that there is $m \in \omega$ such that $[x, W]$ contains $f_{m} \in\left\{f_{n}: n \in \omega\right\}$. This means $x \in U_{m}$. Consequently $\mathcal{U}$ is cover of $X$.

$(2) \Rightarrow(3)$. Let $B_{n} \in \mathcal{B}_{f}$ for each $n \in \omega$. We renumber $\left\{B_{n}\right\}_{n \in \omega}$ as $\left\{B_{i, j}\right\}_{i, j \in \omega}$. Since $B(X)$ is homogeneous, we may think that $f=\mathbf{0}$. We set $\mathcal{U}_{i, j}=\left\{g^{-1}(-1 / i, 1 / i): g \in B_{i, j}\right\}$ for each $i, j \in \omega$. Since $B_{i, j} \in \mathcal{B}_{0}, \mathcal{U}_{i, j}$ is a Baire cover of $X$ for each $i, j \in \omega$. In case the set $M=\left\{i \in \omega: X \in \mathcal{U}_{i, j}\right\}$ is infinite, choose $g_{m} \in B_{m, j} m \in M$ so that $g^{-1}(-1 / m, 1 / m)=X$, then $\left\{g_{m}: m \in \omega\right\} \in \mathcal{B}_{f}$.

So we may assume that there exists $i^{\prime} \in \omega$ such that for each $i \geq i^{\prime}$ and $g \in B_{i, j} g^{-1}(-1 / i, 1 / i)$ is not $X$.

For the sequence $\mathcal{V}_{i}=\left\{\mathcal{U}_{i, j}: j \in \omega\right\}$ of Baire covers there exist $f_{i, j} \in B_{i, j}$ such that $\mathcal{U}_{i}=\left\{f_{i, j}^{-1}(-1 / i, 1 / i): j \in \omega\right\}$ is a cover of $X$. Let $[x, W]$ be any basic open neighborhood of $\mathbf{0}$, where $W=(-\epsilon, \epsilon), \epsilon>0$. There exists $m \geq i^{\prime}$ and $j \in \omega$ such that $1 / m<\epsilon$ and $x \in f_{m, j}^{-1}(-1 / m, 1 / m)$. This means $\left\{f_{i, j}: i, j \in \omega\right\} \in \mathcal{B}_{f}$.

$(3) \Rightarrow(4)$ is immediate.

$(4) \Rightarrow(1)$. Let $A_{n} \in \mathcal{A}$ for each $n \in \omega$. We renumber $\left\{A_{n}\right\}_{n \in \omega}$ as $\left\{A_{i, j}\right\}_{i, j \in \omega}$. Renumber the rational numbers $\mathbb{Q}$ as $\left\{q_{i}: i \in \omega\right\}$. Fix $i \in \omega$. By the assumption there exist $f_{i, j} \in A_{i, j}$ such that $\left\{f_{i, j}: j \in \omega\right\} \in \mathcal{A}_{q_{i}}$ where $q_{i}$ is the constant function to $q_{i}$. Then $\left\{f_{i, j}: i, j \in \omega\right\} \in \mathcal{A}$.

\section{9. $B(X) \models S_{1}(\mathcal{S}, \mathcal{A})$}

Well-known (see in [15]) that $S_{1}\left(\mathcal{B}_{\Gamma}, \mathcal{B}\right) \Leftrightarrow S_{\text {fin }}\left(\mathcal{B}_{\Gamma}, \mathcal{B}\right) \Leftrightarrow U_{\text {fin }}\left(\mathcal{B}_{\Gamma}, \mathcal{B}\right)$.

Theorem 9.1. For a Tychonoff space $X$, the following statements are equivalent:

1. $B(X) \models S_{1}\left(\Gamma_{x}, \mathcal{B}_{f}\right)$;

2. $X \models S_{1}\left(\mathcal{B}_{\Gamma}, \mathcal{B}\right)$. 
Proof. (1) $\Rightarrow(2)$. Let $\left\{\mathcal{U}_{i}\right\} \subset B_{\Gamma}$. For each $i \in \omega$ we consider the set $\mathcal{S}_{i}:=\left\{f \in B(X): f \uparrow U=0\right.$ and $f \uparrow(X \backslash U)=1$ for $\left.U \in \mathcal{U}_{i}\right\}$.

Since $\mathcal{U}_{i}$ is a $\gamma$-cover of $X$, we have that $\mathcal{S}_{i}$ converge to $\mathbf{0}$, i.e. $\mathcal{S}_{i} \in \Gamma_{0}$ for each $i \in \omega$.

Since $B(X) \models S_{1}\left(\Gamma_{x}, \mathcal{B}_{f}\right)$, there is a sequence $\left\{f_{i}\right\}_{i \in \omega}$ such that for each $i, f_{i} \in \mathcal{S}_{i}$, and $\left\{f_{i}: i \in \omega\right\} \in \mathcal{B}_{0}$.

Consider $\mathcal{V}=\left\{U_{i}: U_{i} \in \mathcal{U}_{i}\right.$ such that $f_{i} \uparrow U_{i}=0$ and $\left.f_{i} \uparrow\left(X \backslash U_{i}\right)=1\right\}$. Let $x \in X$ and $W=[x,(-1,1)]$ be a neighborhood of $\mathbf{0}$, then there exists $i_{0} \in \omega$ such that $f_{i_{0}} \in W$.

It follows that $x \in U_{i_{0}}$ and $\mathcal{V} \in \mathcal{B}$. We thus get $X \models S_{1}\left(\mathcal{B}_{\Gamma}, \mathcal{B}\right)$.

$(2) \Rightarrow(1)$. Fix $\left\{S_{n}: n \in \omega\right\} \subset \Gamma_{0}$. We renumber $\left\{S_{n}: n \in \omega\right\}$ as $\left\{S_{i, j}: i, j \in \omega\right\}$.

For each $i, j \in \omega$ and $f \in S_{i, j}$, we put $U_{i, j, f}=\left\{x \in X:|f(x)|<\frac{1}{i+j}\right\}$.

Each $U_{i, j, f}$ is a Baire set of $X$. Let $\mathcal{U}_{i, j}=\left\{U_{i, j, f}: f \in S_{i, j}\right\}$. So without loss of generality, we may assume $U_{i, j, f} \neq X$ for each $i, j \in \omega$ and $f \in S_{i, j}$.

We can easily check that the condition $S_{i, j} \in \Gamma_{0}$ implies that $\mathcal{U}_{i, j}$ is a $\gamma$-cover of $X$.

Since $X \models S_{1}\left(\mathcal{B}_{\Gamma}, \mathcal{B}\right)$ for each $j \in \omega$ there is a sequence $\left\{U_{i, j, f_{i, j}}: i \in \omega\right\}$ such that for each $i, U_{i, j, f_{i, j}} \in \mathcal{U}_{i, j}$, and $\left\{U_{i, j, f_{i, j}}: i \in \omega\right\} \in \mathcal{B}$. Claim that $\left\{f_{i, j}: i, j \in \omega\right\} \in \mathcal{B}_{f}$. Let $x \in X, \epsilon>0$, and $W=[x,(-\epsilon, \epsilon)]$ be a base neighborhood of $\mathbf{0}$, then there exists $j^{\prime} \in \omega$ such that $\frac{1}{1+j^{\prime}}<\epsilon$. It follow that there is $f_{i, j^{\prime}} \in\left\{f_{i, j}: i, j \in \omega\right\}$ such that $f_{i, j^{\prime}}(x) \in(-\epsilon, \epsilon)$.

So $B(X) \models S_{1}\left(\Gamma_{x}, \mathcal{B}_{f}\right)$.

Theorem 9.2. For a space $X$, the following statements are equivalent:

1. $B(X) \models S_{1}(\mathcal{S}, \mathcal{A})$ and is sequentially separable;

2. $X \models S_{1}\left(\mathcal{B}_{\Gamma}, \mathcal{B}\right)$ and $X \models O$;

3. $B(X) \models S_{1}\left(\Gamma_{x}, \mathcal{B}_{f}\right)$ and is sequentially separable;

4. $B(X) \models S_{1}\left(\mathcal{S}, \mathcal{B}_{f}\right)$ and is sequentially separable.

Proof. $(1) \Rightarrow(2)$. Let $\left\{\mathcal{U}_{i}\right\} \subset \mathcal{B}_{\Gamma}$ and $\mathcal{S}=\left\{h_{m}\right\}_{m \in \omega}$ be a countable sequentially dense subset of $B(X)$. Let $\mathcal{U}_{i}=\left\{U_{i}^{m}\right\}_{m \in \omega}$.

For each $i \in \omega$ we consider a countable sequentially dense subset $\mathcal{S}_{i}$ of $B(X)$ where

$\mathcal{S}_{i}=\left\{f_{i}^{m} \in B(X): f_{i}^{m} \uparrow U_{i}^{m}=h_{m}\right.$ and $f_{i}^{m} \uparrow\left(X \backslash U_{i}^{m}\right)=1$ for $\left.m \in \omega\right\}$.

Since $\mathcal{U}_{i}$ is a $\gamma$-cover of $X$ and $\mathcal{S}$ is a countable sequentially dense subset of $B(X)$, we have that $\mathcal{S}_{i}$ is a countable sequentially dense subset of $B(X)$ 
for each $i \in \omega$. Let $h \in B(X)$, there is a sequence $\left\{h_{m_{s}}: s \in \omega\right\} \subset \mathcal{S}$ such that $\left\{h_{m_{s}}\right\}_{s \in \omega}$ converge to $h$. Let $K$ be a finite subset of $X, \epsilon>0$ and $W=<h, K, \epsilon>$ be a base neighborhood of $h$, then there is a number $m_{0}$ such that $K \subset U_{i}^{m}$ for $m>m_{0}$ and $h_{m_{s}} \in W$ for $m_{s}>m_{0}$. Since $f_{i}^{m_{s}}\left\lceil K=h_{m_{s}} \uparrow K\right.$ for each $m_{s}>m_{0}, f_{i}^{m_{s}} \in W$ for each $m_{s}>m_{0}$. It follows that a sequence $\left\{f_{i}^{m_{s}}\right\}_{s \in \omega}$ converge to $h$.

By $B(X) \in S_{1}(\mathcal{S}, \mathcal{A})$, there is a sequence $\left\{f_{i}^{m(i)}\right\}_{i \in \omega}$ such that for each $i$, $f_{i}^{m(i)} \in \mathcal{S}_{i}$, and $\left\{f_{i}^{m(i)}: i \in \omega\right\}$ is an element of $\mathcal{A}$.

Consider a sequence $\left\{U_{i}^{m(i)}: i \in \omega\right\}$.

(a). $U_{i}^{m(i)} \in \mathcal{U}_{i}$.

(b). $\left\{U_{i}^{m(i)}: i \in \omega\right\}$ is a Baire cover of $X$.

Let $K$ be a finite subset of $X$ and $U=<\mathbf{0}, K, \frac{1}{2}>$ be a base neighborhood of $\mathbf{0}$, then there is $f_{i_{j_{0}}}^{m(i)_{j_{0}}} \in U$ for some $j_{0} \in \omega$. It follows that $K \subset U_{i_{j_{0}}}^{m(i)_{j_{0}}}$. We thus get

$X \models S_{1}\left(\mathcal{B}_{\Gamma}, \mathcal{B}\right)$.

$(2) \Leftrightarrow(3)$. By Theorem 9.1 and Theorem 2.4.

(3) $\Rightarrow(4)$ is immediate.

$(4) \Rightarrow(1)$. Suppose that $B(X)$ is sequentially separable and $B(X) \models$ $S_{1}\left(\mathcal{S}, \mathcal{B}_{f}\right)$. Let $D=\left\{d_{n}: n \in \omega\right\}$ be a countable sequentially dense subspace of $B(X)$. Given a sequence of sequentially dense subspace of $B(X)$, enumerate it as $\left\{S_{n, m}: n, m \in \omega\right\}$. For each $n \in \omega$, pick $d_{n, m} \in S_{n, m}$ so that $\left\{d_{n, m}: m \in \omega\right\} \in \mathcal{B}_{d_{n}}$. Then $\left\{d_{n, m}: m, n \in \omega\right\} \in \mathcal{A}$.

We can summarize the relationships between considered notions in next diagrams. 


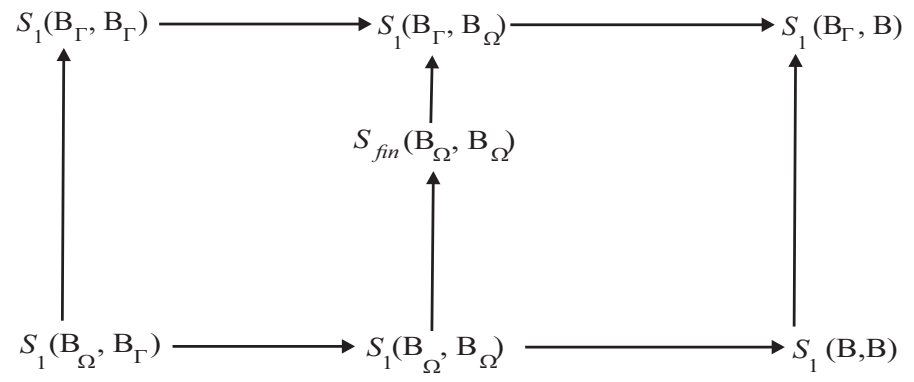

Figure 2. The Scheepers Diagram in the Baire case.

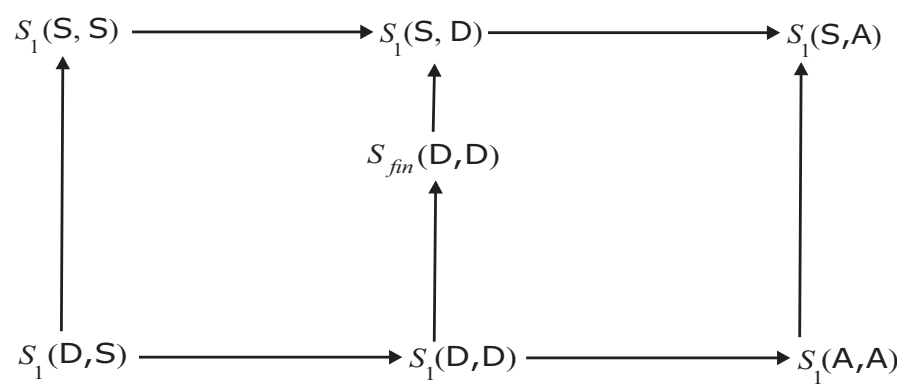

Figure 3. The Diagram of selection principles of selectors for sequences of countable dense and countable sequentially subsets of $B(X)$ corresponding to selection principles of Figure 2.

\section{Critical cardinalities}

The critical cardinalities in the Scheepers Diagram in the Baire case are equal to the critical cardinalities of selectors for sequences of countable dense and countable sequentially subsets of $B(X)$.

- According to Corollary 41 in [15], Theorem 3.2 and Theorem 7.2 it is consistent that there is a set of real numbers $X$ such that $B(X)$ with property $S_{1}(\mathcal{D}, \mathcal{D})$, but not property $S_{1}(\mathcal{S}, \mathcal{S})$.

- According to Theorem 32 in [15], Theorem 8.3 and Theorem 6.2 it is consistent that there is a set of real numbers $X$ such that $B(X)$ in $S_{1}(\mathcal{A}, \mathcal{A})$ which is not in $S_{1}(\mathcal{S}, \mathcal{D})$.

- According to Theorem 43 in [15], Theorem 8.3, Theorem 3.2 and Theorem 7.2 it is consistent that there is a set of real numbers $X$ such that $B(X)$ in $S_{1}(\mathcal{S}, \mathcal{S})$ and not in either of $S_{\text {fin }}(\mathcal{D}, \mathcal{D})$ or $S_{1}(\mathcal{A}, \mathcal{A})$. 
- According to Theorem 27 in [15] and Theorem 4.2 the minimal cardinality of a set of real numbers $X$ such that $B(X)$ not having property $S_{f i n}(\mathcal{D}, \mathcal{D})$ is $\mathfrak{d}$ while the minimal cardinality of a set of real numbers $X$ such that $B(X)$ not having property $S_{1}(\mathcal{A}, \mathcal{A})$ is $\operatorname{cov}(\mathcal{M})$. Since it is consistent that $\operatorname{cov}(\mathcal{M})<\mathfrak{d}$, it is consistent that none of the arrows starting at the bottom row of Fig. 3 is reversible.

For a collection $\mathcal{J}$ of spaces of all Baire functions, defined on Tychonoff spaces $X$ with $i w(X)=\aleph_{0}$, let $\operatorname{non} B(\mathcal{J})$ denote the minimal cardinality for $X$ which $B(X)$ is not a member of $\mathcal{J}$.

Theorem 10.1. For a collection $B(X)$ of spaces of all Baire functions, defined on Tychonoff spaces $X$ with iw $(X)=\aleph_{0}$,

(1) $\operatorname{non} B\left(S_{1}(\mathcal{D}, \mathcal{S})\right)=\mathfrak{p}$.

(2) $\operatorname{non} B\left(S_{1}(\mathcal{S}, \mathcal{S})\right)=\mathfrak{b}$.

(3) $\operatorname{non} B\left(S_{\text {fin }}(\mathcal{D}, \mathcal{D})\right)=\operatorname{non} B\left(S_{1}(\mathcal{S}, \mathcal{D})\right)=\operatorname{non} B\left(S_{1}(\mathcal{S}, \mathcal{A})\right)=\mathfrak{d}$.

(4) $\operatorname{non} B\left(S_{1}(\mathcal{D}, \mathcal{D})\right)=\operatorname{non} B\left(S_{1}(\mathcal{A}, \mathcal{A})\right)=\operatorname{cov}(\mathcal{M})$.

Proof. By Theorem 27 [15],

(1) $\operatorname{non}\left(S_{1}\left(\mathcal{B}_{\Omega}, \mathcal{B}_{\Gamma}\right)\right)=\mathfrak{p}$. By Theorem 5.2, $\operatorname{non} B\left(S_{1}(\mathcal{D}, \mathcal{S})\right)=\mathfrak{p}$.

(2) $\operatorname{non}\left(S_{1}\left(\mathcal{B}_{\Gamma}, \mathcal{B}_{\Gamma}\right)\right)=\mathfrak{b}$. By Theorem 7.2 , $\operatorname{non} B\left(S_{1}(\mathcal{S}, \mathcal{S})\right)=\mathfrak{b}$.

(3) $\operatorname{non}\left(S_{\text {fin }}\left(\mathcal{B}_{\Omega}, \mathcal{B}_{\Omega}\right)\right)=\mathfrak{d}$. By Theorem 4.2, $\operatorname{non} B\left(S_{\text {fin }}(\mathcal{D}, \mathcal{D})\right)=\mathfrak{d}$.

(4) non $\left(S_{1}\left(\mathcal{B}_{\Gamma}, \mathcal{B}_{\Omega}\right)\right)=\mathfrak{d}$. By Theorem 6.2, $\operatorname{non} B\left(S_{1}(\mathcal{S}, \mathcal{D})\right)=\mathfrak{d}$.

$(5) \operatorname{non}\left(S_{1}\left(\mathcal{B}_{\Gamma}, \mathcal{B}\right)\right)=\mathfrak{d}$. By Theorem 9.2, $\operatorname{non} B\left(S_{1}(\mathcal{S}, \mathcal{A})\right)=\mathfrak{d}$.

(6) $\operatorname{non}\left(S_{1}\left(\mathcal{B}_{\Omega}, \mathcal{B}_{\Omega}\right)\right)=\operatorname{cov}(\mathcal{M})$.

By Theorem 3.2, $\operatorname{non} B\left(S_{1}(\mathcal{D}, \mathcal{D})\right)=\operatorname{cov}(\mathcal{M})$.

(7) $\operatorname{non}\left(S_{1}(\mathcal{B}, \mathcal{B})\right)=\operatorname{cov}(\mathcal{M})$.

By Theorem 8.3, $\operatorname{non} B\left(S_{1}(\mathcal{A}, \mathcal{A})\right)=\operatorname{cov}(\mathcal{M})$.

\section{References}

[1] A.V. Arhangel'skii, The frequency spectrum of a topological space and the classification of spaces, Soviet Math. Dokl. 13, (1972), 1186-1189.

[2] A.V. Arhangelskii, Hurewicz spaces, analytic sets and fan tightness of function spaces, Soviet Math. Dokl. 33, (1986), 396-399.

[3] A. Bella, M. Bonanzinga, M. Matveev, Variations of selective separability, Topology and its Applications, 156, (2009), 1241-1252. 
[4] L. Bukovský, J. Šupina, Sequence selection principles for quasi-normal convergence, Topology and its Applications, 159, (2012), p.283-289.

[5] E.K. van Douwen, The integers and topology, in: Handbook of SetTheoretic Topology, North-Holland, Amsterdam, (1984).

[6] W. Just, A.W. Miller, M. Scheepers, P.J. Szeptycki, The combinatorics of open covers, II, Topology and its Applications, 73, (1996), 241-266.

[7] N. Noble, The density character of functions spaces, Proc. Amer. Math. Soc. (1974), V.42, is.I.-P., 228-233.

[8] A.V. Osipov, The application of selection principles in the study of the properties of function spaces, to appear.

[9] A.V. Osipov, Classification of selectors for sequences of dense sets of $C_{p}(X)$, to appear.

[10] Strongly sequentially separable function spaces, via selection principles, to appear.

[11] A.V. Pestrjkov, O prostranstvah berovskih funktsii, Issledovanij po teorii vipuklih mnogestv i grafov, Sbornik nauchnih trudov, Sverdlovsk, Ural'skii Nauchnii Center, (1987), p.53-59.

[12] M. Sakai, Property $C^{\prime \prime}$ and function spaces, Proc. Amer. Math. Soc. 104 (1988), 917919.

[13] M. Sakai, M. Scheepers, The combinatorics of open covers, Recent Progress in General Topology III, (2013), Chapter, p. 751-799.

[14] M. Scheepers, A sequential property of $C_{p}(X)$ and a covering property of Hurewicz, Proceedings of the American Mathematical Society, 125, (1997), p. 2789-2795.

[15] M. Scheepers, B. Tsaban, The combinatorics of Borel covers, Topology and its Applications, 121, (2002), p.357-382.

[16] B. Tsaban, L. Zdomskyy, Hereditarily Hurewicz spaces and Arhangel'skï sheaf amalgamations, Journal of the European Mathematical Society, 12, (2012), 353-372. 